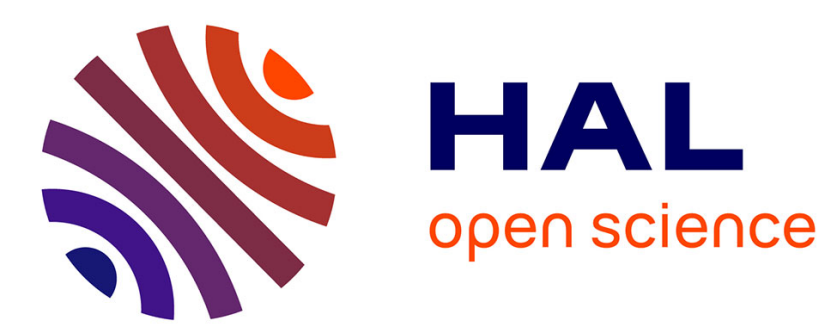

\title{
Prise en charge des voies aériennes supérieures au cours de l'anesthésie générale en fin de travail : étude multicentrique rétrospective
}

Maya Enser

\section{To cite this version:}

Maya Enser. Prise en charge des voies aériennes supérieures au cours de l'anesthésie générale en fin de travail : étude multicentrique rétrospective. Médecine humaine et pathologie. 2014. dumas-00967402

\section{HAL Id: dumas-00967402 https://dumas.ccsd.cnrs.fr/dumas-00967402}

Submitted on 28 Mar 2014

HAL is a multi-disciplinary open access archive for the deposit and dissemination of scientific research documents, whether they are published or not. The documents may come from teaching and research institutions in France or abroad, or from public or private research centers.
L'archive ouverte pluridisciplinaire HAL, est destinée au dépôt et à la diffusion de documents scientifiques de niveau recherche, publiés ou non, émanant des établissements d'enseignement et de recherche français ou étrangers, des laboratoires publics ou privés. 
FACULTE MIXTE DE MEDECINE ET DE PHARMACIE DE ROUEN

\title{
THESE POUR LE DOCTORAT EN MEDECINE
}

(Diplôme d'Etat)

\author{
PAR \\ Maya ENSER
}

Née le 12 mai 1983 à Paris $14^{\text {ème }}$

Présentée et soutenue publiquement le 14 février 2014

PRISE EN CHARGE DES VOIES AERIENNES SUPERIEURE AU COURS DE L'ANESTHESIE GENERALE EN FIN DE TRAVAIL : ETUDE MULTICENTRIQUE RETROSPECTIVE

PRESIDENT DE JURY : Monsieur le Professeur Bertrand DUREUIL DIRECTEUR DE THESE : Monsieur le Docteur Fabien TOURREL 
DOYEN :

ASSESSEURS :

DOYENS HONORAIRES :

PROFESSEURS HONORAIRES :
Professeur Pierre FREGER

Professeur Michel GUERBET

Professeur Benoit VEBER

Professeur Pascal JOLY

Professeurs J. BORDE - Ph. LAURET - H. PIGUET - C. THUILLEZ

MM. M-P AUGUSTIN - J.ANDRIEU-GUITRANCOURT - M.BENOZIO-

J.BORDE - Ph. BRASSEUR - R. COLIN - E. COMOY - J. DALION - DESHAYES C. FESSARD - J.P FILLASTRE - P.FRIGOT -J. GARNIER - J. HEMET - B. HILLEMAND - G. HUMBERT - J.M. JOUANY - R. LAUMONIER - Ph. LAURET - M. LE FUR - J.P. LEMERCIER - J.P LEMOINE - MIE MAGARD - MM. B. MAITROT M. MAISONNET - F. MATRAY - P.MITROFANOFF - MME A. M. ORECCHIONI - P. PASQUIS - H.PIGUET - M.SAMSON - MmE SAMSON-DOLLFUS - J.C. SCHRUB

- R.SOYER - B.TARDIF - TESTART - J.M. THOMINE - C. THUILLEZ - P.TRON C.WINCKLER - L.M.WOLF

\section{I- MEDECINE}

\section{PROFESSEURS}

\section{Frẻdéric ANSELME}

Mme Isabelle AUQUIT AUCKBUR

M. Bruno BACHY (Surnombre)

M. Fabrice BAUER

Mme Soumeya BEKRI

M. Jacques BENICHOU

M. Jean-Paul BESSOU

Mme Françoise BEURET-BLANQUART (Surnombre)

M. GUY BONMARCHAND

M. Olivier BOYER

M. Jean-François CAILLARD (Surnombre)

M. François CARON

M. Philippe CHASSAGNE

$M$. Vincent COMPERE

M. Antoine CUVELIER

M. Pierre CZERNICHOW

M. Jean - Nicolas DACHER

M. Stéfan DARMONI

M. Pierre DECHELOTTE

$\begin{array}{ll}\text { HCN } & \text { Cardiologie } \\ \mathrm{HCN} & \text { Chirurgie Plastique } \\ \mathrm{HCN} & \text { Chirurgie pédiatrique } \\ \mathrm{HCN} & \text { Cardiologie } \\ \mathrm{HCN} & \text { Biochimie et Biologie Moléculaire } \\ \mathrm{HCN} & \text { Biostatistiques et informatique médicale } \\ \mathrm{HCN} & \text { Chirurgie thoracique et cardio-vasculaire } \\ \mathrm{CRMPR} & \text { Médecine physique et de réadaptation } \\ \mathrm{HCN} & \text { Réanimation médicale } \\ \mathrm{UFR} & \text { Immunologie } \\ \mathrm{HCN} & \text { Médecine et santé au Travail } \\ \mathrm{HCN} & \text { Maladies infectieuses et tropicales } \\ \mathrm{HB} & \text { Médecine interne (Gériatrie) } \\ \mathrm{HCN} & \text { Anesthésiologie et réanimation chirurgicale } \\ \mathrm{HB} & \text { Pneumologie } \\ \mathrm{HCH} & \text { Epidémiologie, économie de la santé } \\ \mathrm{HCN} & \text { Radiologie et Imagerie Médicale } \\ \mathrm{HCN} & \text { Informatique Médicale/Techniques de communication } \\ \mathrm{HCN} & \text { Nutrition }\end{array}$




\begin{tabular}{|c|c|c|}
\hline Mme Danièle DEHESDIN (Surnombre) & $\mathrm{HCN}$ & Oto-Rhino-Laryngologie \\
\hline M. Jean DOUCET & HB & Thérapeutique/Médecine - Interne - Gériatrie. \\
\hline M. Bernard DUBRAY & $\mathrm{CB}$ & Radiothérapie \\
\hline M. Philippe DUCROTTE & $\mathrm{HCN}$ & Hépato - Gastro - Entérologie \\
\hline M. Frank DUJARDIN & $\mathrm{HCN}$ & Chirurgie Orthopédique - Traumatologique \\
\hline M. Fabrice DUPARC & $\mathrm{HCN}$ & Anatomie - Chirurgie Orthopédique et Traumatologique \\
\hline M. Bertrand DUREUIL & $\mathrm{HCN}$ & Anesthésiologie et réanimation chirurgicale \\
\hline Mme Hélène ELTCHANINOFF & $\mathrm{HCN}$ & Cardiologie \\
\hline M. Thierry FREBOURG & UFR & Génétique \\
\hline M. Pierre FREGER & $\mathrm{HCN}$ & Anatomie/Neurochirurgie \\
\hline M. Jean François GEHANNO & $\mathrm{HCN}$ & Médecine et Santé au Travail \\
\hline M. Emmanuel GERARDIN & $\mathrm{HCN}$ & Imagerie Médicale \\
\hline Mme Priscille GERARDIN & $\mathrm{HCN}$ & Pédopsychiatrie \\
\hline M. Michel GODIN & $\mathrm{HB}$ & Néphrologie \\
\hline M. Guillaume GOURCEROL & $\mathrm{HCN}$ & Physiologie \\
\hline M. Philippe GRISE & $\mathrm{HCN}$ & Urologie \\
\hline M. Didier HANNEQUIN & $\mathrm{HCN}$ & Neurologie \\
\hline M. Fabrice JARDIN & $\mathrm{CB}$ & Hématologie \\
\hline M. Luc-Marie JOLY & $\mathrm{HCN}$ & Médecine d'urgence \\
\hline M. Pascal JOLY & $\mathrm{HCN}$ & Dermato - vénéréologie \\
\hline M. Jean-Marc KUHN & $\mathrm{HB}$ & Endocrinologie et maladies métaboliques \\
\hline Mme Annie LAQUERRIERE & $\mathrm{HCN}$ & Anatomie cytologie pathologiques \\
\hline M. Vincent LAUDENBACH & $\mathrm{HCN}$ & Anesthésie et réanimation chirurgicale \\
\hline M. JoëI LECHEVALLIER & $\mathrm{HCN}$ & Chirurgie infantile \\
\hline M. Hervé LEFEBVRE & $\mathrm{HB}$ & Endocrinologie et maladies métaboliques \\
\hline M. Thierry LEQUERRE & $\mathrm{HB}$ & Rhumatologie \\
\hline M. Eric LEREBOURS & $\mathrm{HCN}$ & Nutrition \\
\hline Mme Anne-Marie LEROI & $\mathrm{HCN}$ & Physiologie \\
\hline M. Hervé LEVESQUE & $\mathrm{HB}$ & Médecine interne \\
\hline Mme Agnès LIARD-ZMUDA & $\mathrm{HCN}$ & Chirurgie Infantile \\
\hline M. Pierre Yves LITZLER & $\mathrm{HCN}$ & Chirurgie Cardiaque \\
\hline M. Bertrand MACE & $\mathrm{HCN}$ & Histologie, embryologie, cytogénétique \\
\hline M. David MALTETE & $\mathrm{HCN}$ & Neurologie \\
\hline M. Christophe MARGUET & $\mathrm{HCN}$ & Pédiatrie \\
\hline Mme Isabelle MARIE & HB & Médecine Interne \\
\hline M. Jean-Paul MARIE & $\mathrm{HCN}$ & ORL \\
\hline M. LOİC MARPEAU & $\mathrm{HCN}$ & Gynécologie - obstêtrique \\
\hline M. Stéphane MARRET & $\mathrm{HCN}$ & Pédiatrie \\
\hline Mme Vẻronique MERLE & $\mathrm{HCN}$ & Epidémiologie \\
\hline M. Pierre MICHEL & $\mathrm{HCN}$ & Hépato - Gastro - Entérologie \\
\hline M. Francis MICHOT & $\mathrm{HCN}$ & Chirurgie digestive \\
\hline M. Bruno MIHOUT (Surnombre) & $\mathrm{HCN}$ & Neurologie \\
\hline M. Jean-François MUIR & HB & Pneumologie \\
\hline
\end{tabular}




\author{
M. Marc MURAINE \\ M. Philippe MUSETtE \\ M. Christophe PEILLON \\ M. Jean-Marc PERON \\ M. Christian PFISTER \\ M. Jean-Christophe PLANTIER \\ M. Didier PLISSONNIER \\ M. Bernard PROUST \\ M. François PROUST \\ Mme Nathalie RIVES \\ M. Jean-Christophe RICHARD (Mise en dispo) \\ M. Horace ROMAN \\ M. Jean-Christophe SABOURIN \\ M. Guillaume SAVOYE \\ Mme Céline SAVOYE - COLLET \\ Mme Pascale SCHNEIDER \\ M. Michel SCOTTE \\ Mme Fabienne TAMION \\ Mme Florence THIBAUT \\ M. LUC THIBERVILLE \\ M. Christian THUILLEZ \\ M. Hervé TILLLY \\ M. François TRON (Surnombre) \\ M. Jean-Jacques TUECH \\ M. Jean-Pierre VANNIER \\ M. Benoît VEber \\ M. Pierre VERA \\ M. Eric VERIN \\ M. ERIC VERSPYCK \\ M. Olivier VITTECOQ \\ M. Jacques WEBER
}

\section{MAITRES DE CONFERENCES}

Mme Noëlle BARBIER-FREBOURG

M. JeremY BELLIEN

Mme Carole BRASSE LAGNEL

M. Gérard BUCHONNET

Mme Mireille CASTANET

Mme Nathalie CHASTAN

Mme Sophie CLAEYSSENS

M. Moise COEfFIER

M. Stéphane DERREY

\begin{tabular}{ll}
$\mathrm{HCN}$ & Ophtalmologie \\
$\mathrm{HCN}$ & Dermatologie-Vénéréologie \\
$\mathrm{HCN}$ & Chirurgie générale \\
$\mathrm{HCN}$ & Stomatologie et chirurgie maxillo-faciale \\
$\mathrm{HCN}$ & Urologie \\
$\mathrm{HCN}$ & Bactériologie - Virologie \\
$\mathrm{HCN}$ & Chirurgie vasculaire \\
$\mathrm{HCN}$ & Médecine légale \\
$\mathrm{HCN}$ & Neurochirurgie \\
$\mathrm{HCN}$ & Biologie et méd. du dévelop. et de la reprod. \\
$\mathrm{HCN}$ & Réanimation Médicale, Médecine d'urgence \\
$\mathrm{HCN}$ & Gynécologie Obstétrique \\
$\mathrm{HCN}$ & Anatomie - Pathologie \\
$\mathrm{HCN}$ & Hépato-Gastro \\
$\mathrm{HCN}$ & Imagerie Médicale \\
$\mathrm{HCN}$ & Pédiatrie \\
$\mathrm{HCN}$ & Chirurgie digestive \\
$\mathrm{HCN}$ & Thérapeutique \\
$\mathrm{HCN}$ & Psychiatrie d'adultes \\
$\mathrm{HCN}$ & Pneumologie \\
$\mathrm{HB}$ & Pharmacologie \\
$\mathrm{CB}$ & Hématologie et transfusion \\
$\mathrm{UFR}$ & Immunologie \\
$\mathrm{HCN}$ & Chirurgie digestive \\
$\mathrm{HCN}$ & Pédiatrie génétique \\
$\mathrm{HCN}$ & Anesthésiologie Réanimation chirurgicale \\
$\mathrm{C.B}$ & Biophysique et traitement de limage \\
$\mathrm{CRMPR}$ & Médecine physique et de réadaptation \\
$\mathrm{HCN}$ & Gynécologie obstétrique \\
$\mathrm{HB}$ & Rhumatologie \\
& Physiologie \\
\hline $\mathrm{HCN}$ &
\end{tabular}

$\begin{array}{ll}\mathrm{HCN} & \text { Bactériologie - Virologie } \\ \mathrm{HCN} & \text { Pharmacologie } \\ \mathrm{HCN} & \text { Biochimie } \\ \mathrm{HCN} & \text { Hématologie } \\ \mathrm{HCN} & \text { Pédiatrie } \\ \mathrm{HCN} & \text { Physiologie } \\ \mathrm{HCN} & \text { Biochimie et biologie moléculaire } \\ \mathrm{HCN} & \text { Nutrition } \\ \mathrm{HCN} & \text { Neurochirurgie }\end{array}$


M. ERIC DURAND

M. Manuel ETIENNE

M. Serge JACQUOT

M. Joël LADNER

M. Jean-Baptiste LATOUCHE

M. Thomas MOUREZ

M. Jean-François MENARD

Mme Muriel QUILLARD

M. Vincent RICHARD

M. Francis ROUSSEL

Mme Pascale SAUGIER-VEBER

Mme Anne-Claire TOBENAS-DUJARDIN

M. Pierre Hugues VIVIER

\section{PROFESSEUR AGREGE OU CERTIFIE}

Mme Dominique LANIEZ

Mme Cristina BADULESCU
$\mathrm{HCN} \quad$ Cardiologie

$\mathrm{HCN} \quad$ Maladies infectieuses et tropicales

UFR Immunologie

$\mathrm{HCN} \quad$ Epidémiologie, économie de la santé

UFR Biologie Cellulaire

$\mathrm{HCN}$ Bactériologie

$\mathrm{HCN}$ Biophysique

$\mathrm{HCN} \quad$ Biochimie et Biologie moléculaire

UFR Pharmacologie

$\mathrm{HCN}$ Histologie, embryologie, cytogénétique

$\mathrm{HCN}$ Génétique

$\mathrm{HCN} \quad$ Anatomie

$\mathrm{HCN} \quad$ Imagerie Médicale

UFR Anglais

UFR Communication 


\title{
II - PHARMACIE
}

\section{PROFESSEURS}

\author{
M. Thierry BESSON \\ M. Jean-Jacques BONNET \\ M. Roland CAPRON (PU-PH) \\ M. Jean COSTENTIN (Professeur émérite) \\ Mme Isabelle DUBUS \\ M. LoĪ́ FAVENNEC (PU-PH) \\ M. Jean Pierre GOULLE \\ M. Michel GUERBET \\ M. Olivier LAFONT \\ Mme Isabelle LEROUX \\ M. Paul MULDER \\ Mme Martine PESTEL-CARON (PU-PH) \\ Mme Elisabeth SEGUIN \\ M. Rèmi VARIN (PU-PH) \\ $M$ Jean-Marie VAUGEOIS \\ M. Philippe VERITE
}

\section{MAITRES DE CONFERENCES}

Mme Cécile BARBOT
Mme Dominique BOUCHER
M. Frédéric BOUNOURE
M. Abdeslam CHAGRAOUI
M. Jean CHASTANG
Mme Marie Catherine CONCE-CHEMTOB
Mme Elizabeth CHOSSON
Mme Cécile CORBIERE
M. Eric DITTMAR
Mme Nathalie DOURMAP
Mme Isabelle DUBUC
M. Abdelhakim ELOMRI
M. François ESTOUR
M. Gilles GARGALA (MCU-PH)
Mme Najla GHARBI
Mme Marie-Laure GROULT
M. Hervé HUE
Mme Laetitia LE GOFF
Mme Hong LU

\author{
Chimie Thérapeutique \\ Pharmacologie \\ Biophysique \\ Pharmacologie \\ Biochimie \\ Parasitologie \\ Toxicologie \\ Toxicologie \\ Chimie organique \\ Physiologie \\ Sciences du médicament \\ Microbiologie \\ Pharmacognosie \\ Pharmacie Hospitalière \\ Pharmacologie \\ Chimie analytique
}

\author{
Chimie Générale et Minérale \\ Pharmacologie \\ Pharmacie Galénique \\ Physiologie \\ Biomathématiques \\ Législation pharmaceutique et économie de la santé \\ Botanique \\ Biochimie \\ Biophysique \\ Pharmacologie \\ Pharmacologie \\ Pharmacognosie \\ Chimie Organique \\ Parasitologie \\ Chimie analytique \\ Botanique \\ Biophysique et Mathématiques \\ Parasitologie Immunologie \\ Biologie
}


Mme Sabine MENAGER

Mme Christelle MONTEIL

M. Mohamed SKIBA

Mme Malika SKIBA

Mme Christine THARASSE

$M$. Frédéric ZIEGLER

\section{PROFESSEUR CONTRACTUEL}

Mme Elizabeth DE PAOLIS

Anglais

\section{ATTACHE TEMPORAIRE D'ENSEIGNEMENT ET DE RECHERCHE}

M. Imane EL MEOUCHE

Bactériologie

Mme Juliette GAUTIER

M. Romy RAZAKANDRAINIBE

Galénique

Parasitologie
Chimie organique

Toxicologie

Pharmacie Galénique

Pharmacie Galénique

Chimie thérapeutique

Biochimie 


\section{PROFESSEURS}

M. Jean-Loup HERMIL

UFR Médecine générale

\section{PROFESSEURS ASSOCIES A MI-TEMPS}

M. Pierre FAINSILBER

UFR Médecine générale

M. Alain MERCIER

UFR Médecine générale

M. Philippe NGUYEN THANH

UFR Médecine générale

\section{MAITRE DE CONFERENCES ASSOCIE A MI-TEMPS}

$\begin{array}{lll}\text { M Emmanuel LEFEBVRE } & \text { UFR } & \text { Médecine générale } \\ \text { Mme Elisabeth MAUVIARD } & \text { UFR } & \text { Médecine générale } \\ \text { Mme Marie Thérèse THUEUX } & \text { UFR } & \text { Médecine générale } \\ \text { Mme Yveline SERVIN } & \text { UFR } & \text { Médecine Générale }\end{array}$

CHEF DES SERVICES ADMINISTRATIFS : Mme Véronique DELAFONTAINE 
Mme Cécile BARBOT

M. Thierry BESSON

M. Roland CAPRON

M Jean CHASTANG

Mme Marie-Catherine CONCE-CHEMTOB

Mme Elisabeth CHOSSON

M. Jean-Jacques BONNET

Mme Isabelle DUBUS

M. LoĪ́ FAVENNEC

M. Michel GUERBET

M. Olivier LAFONT

Mme Isabelle LEROUX-NICOLLET

Mme Martine PESTEL-CARON

Mme Elisabeth SEGUIN

M. Mohamed SKIBA

M. Philippe VERITE
Chimie Générale et Minêrale

Chimie thérapeutique

Biophysique

Mathématiques

Législation, Economie de la Santé

Botanique

Pharmacodynamie

Biochimie

Parasitologie

Toxicologie

Chimie organique

Physiologie

Microbiologie

Pharmacognosie

Pharmacie Galénique

Chimie analytique 


\section{MAITRES DE CONFERENCES}

M. Sahil ADRIOUCH

Biochimie et biologie moléculaire (Unité Inserm 905)

Mme Gaëlle BOUGEARD-DENOYELLE

Biochimie et biologie moléculaire (UMR 1079)

Mme Carine CLEREN

Neurosciences (Néovasc)

Mme Pascaline GAILDRAT

Génétique moléculaire humaine (UMR 1079)

M. Antoine OUVRARD-PASCAUD

Physiologie (Unité Inserm 1076)

Mme Isabelle TOURNIER

Biochimie (UMR 1079)

\section{PROFESSEURS DES UNIVERSITES}

M. Serguei FETISSOV

Physiologie (Groupe ADEN)

Mme Su RUAN

Génie Informatique 
Par délibération en date du 3 mars 1967, la faculté a arrêté que les opinions émises dans les dissertations qui lui seront présentées doivent être considérées comme propres à leurs auteurs et qu'elle n'entend leur donner aucune approbation ni improbation. 
A Monsieur le Professeur Bertand Dureuil, pour m'avoir offert une formation rigoureuse à l'anesthésie-réanimation. Pour m'avoir fait l'honneur de juger ce travail et de présider ce jury.

A Monsieur le Professeur Vincent Compère, pour son coaching personnalisé et pour toutes les séances de soutien et de réassurance dans son bureau, ou en garde entre 2 péri (ou 2 AG !), pour le temps et l'énergie consacrés à nous former tout en restant disponible, à l'écoute et bienveillant. Pour m'avoir fait l'honneur d'accepter de juger ce travail.

A Monsieur le Professeur Eric Verspyck, pour avoir apporté son regard de gynécologue-obstétricien sur ce sujet. Pour m'avoir fait l'honneur d'accepter de juger ce travail.

A Monsieur le Docteur Fabien Tourrel, sans qui le chapitre Rouennais de ma vie n'aurait pas été le même : pour son optimisme, sa gentillesse, sa disponibilité et son soutien pour la rédaction de cette thèse, son humour, ses talents culinaires et musicaux, son légendaire esprit de compèt', et évidemment, pour m'avoir invité, l'air de rien, un certain lundi soir de mai 2011 à un certain diner rue aux Ours... Pour m'avoir fait l'honneur d'accepter de diriger ce travail. 
A ma famille,

Mes parents, pour les valeurs qu'ils m'ont transmises et pour leur soutien inconditionnel.

Mes sœurs, mes deux indispensables piliers: Sarah, pour avoir été (et être encore) un si bel exemple, et Noémie, pour m'avoir donné le goût de prendre soin des autres et pour nos fousrires.

Mes grands-parents, présent et absents, pour tout leur amour.

Mon oncle Gilou pour m'avoir autant soutenu pendant mes études, et à Mimi.

Raphaël et Arthur, mes adorables beaux-frères.

Léa, pour sa super-mignonneté.

A Clément, pour être exactement celui qu'il me fallait.

A Christiane et Philippe, Emilie, Marie, Jehanne et Akim, pour leur accueil si chaleureux. A Ladislas et Pierre, pour leur grande bienveillance.

A mes amis parisiens,

Nina, pour sa sagesse exemplaire.

Alice, pour ces longues années d'études de médecine vécues main dans la main, du début à la fin, de Paris à Rouen, et aussi pour son amitié infaillible...

Chloé $\mathrm{S}$, pour être une si fervente supportrice (je crois que la thèse était le dernier événement-clé de mes études à noter dans ton agenda !).

Camille, pour sa joie de vivre communicative.

Chloé $\mathrm{D}$, pour ce fil tellement précieux.

Joy, pour nos 25 ans d'amitié.

Anneso, pour nos sous-colles et tout ce qui a suivi.

Flauby, pour être si drôle.

A mes amis rouennais,

Sinouche, pour son humour, ses délicieux sinouccini, ses astuces forme-et-minceur, son oreille attentive et ses conseils avisés. Votez pour lui !

Middy, pour sa sérénité, son pragmatisme et sa capacité à relativiser.

Mes autres co-internes: Audrey pour sa spontanéité, JB pour son altruisme, Thomas pour nos gouts professionnels communs, Paul pour sa constance, Alex pour son professionnalisme, Antoine pour les trajets en voiture du début, Yaya pour son calme, Edouard pour ses blagues salasses, et tous ceux que je ne cite pas... 
Anne-Lise, pour ses incroyables gâteaux et sa sensibilité.

Steven, pour supporter avec bonne humeur mes râleries, pour avoir failli courir avec moi les 20 $\mathrm{km}$.

Romain, pour tous les trajets Paris-Rouen passés à refaire le monde, en apprenant à conduire.

Mélanie et Mathieu, pour leur gentillesse.

Nimrod et Virginie, pour nos seder et nos noelnouka à la rouennaise.

Claire, pour son humour.

Eric et Caroline, pour ce fameux diner de mai 2011 (et tous ceux qui ont suivi !).

Manu, pour ses taquineries.

Schéché, pour ses barbeuc' et pour son accueil chaleureux à mon arrivée à Rouen.

Aux médecins que j'ai rencontrés au fil de mon internat et à qui j'espère ressembler un jour :

Olivier, pour sa logique implacable, son humour, et pour toutes ses nuits passées sur le parking des Feugrais..., Fabienne pour ses conseils si précieux et pour ses schémas récapitulatifs en pleine CV (celle de $3 \mathrm{~h}$ du matin), Christophe, pour sa montagne de connaissances, Jean-Philippe pour sa rigueur et son humanité, et Maamar pour son calme résistant à toute épreuve.

Aux équipes médicales et paramédicales du bloc uro-dig, de la réanimation chirurgicale, de la réanimation cardiaque, de la réanimation médicale, de la réanimation dieppoise, et de la réanimation elbeuvienne, pour leur accueil chaleureux, et pour tout ce que j'y ai appris, médicalement et humainement.

A ceux qui ont contribué à la réalisation de cette thèse : le Professeur Horace Roman et le docteur Dominique Guerrot pour les 12 versions de l'analyse multivariée, le docteur Samuel Leroy pour m'avoir ouvert les portes du Belvédère, et les archivistes. 
Abréviations

1 Introduction

1.1 Modifications anatomiques et physiologiques liées à la grossesse ................................... 2

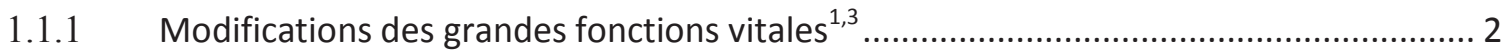

1.1.2 Modifications liées à certains terrains ou états pathologiques ................................... 4

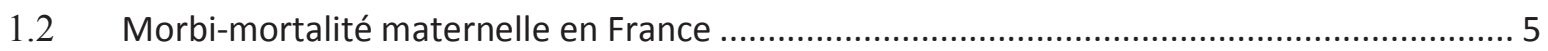

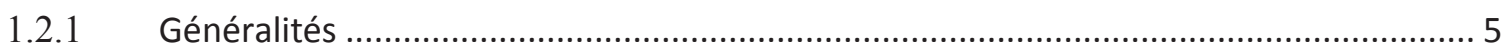

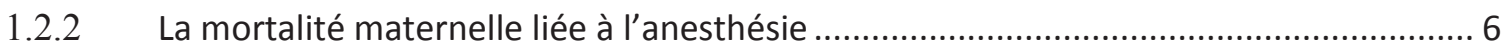

1.2.3 Les principales complications de l’anesthésie générale en obstétrique .......................... 6

1.3 Evaluation des voies aériennes supérieures en obstétrique.............................................. 9

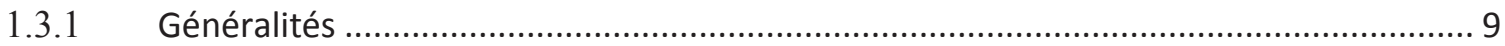

1.3.2 Particularités de l'évaluation des voies aériennes supérieures en obstétrique ............ 10

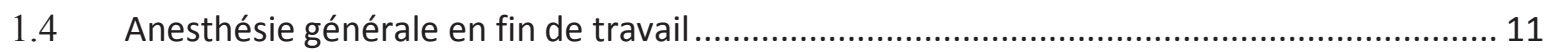

1.4.1 Evolution des pratiques en obstétrique et en anesthésie obstétricale ....................... 11

1.4.2 Indications de l'anesthésie générale en salle de naissance ....................................... 12

1.4.3 Prévention du syndrome de Mendelson ............................................................. 12

1.4.4 Anesthésie générale en salle de naissance et gestion des voies aériennes.................. 14

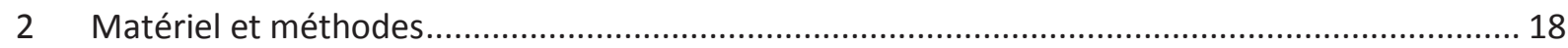

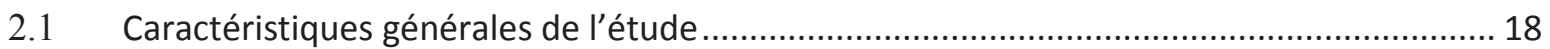

$2.2 \quad$ Objectifs de l'étude et critères de jugement.................................................................... 18

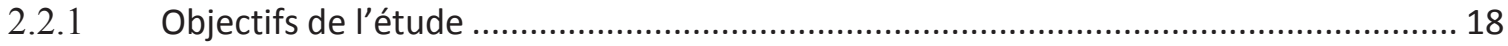

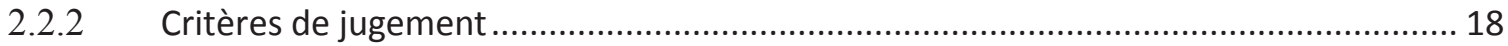

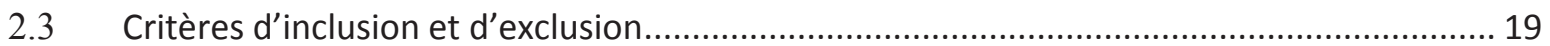

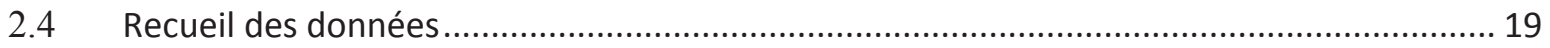

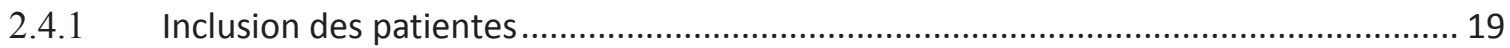

2.4.2 Elaboration du tableau de recueil de données ........................................................ 20 
Sources des données ................................................................................... 20

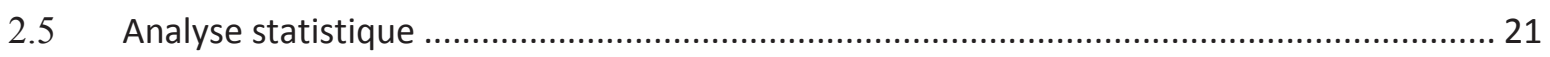

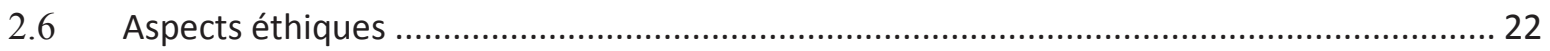

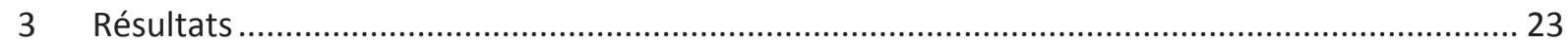

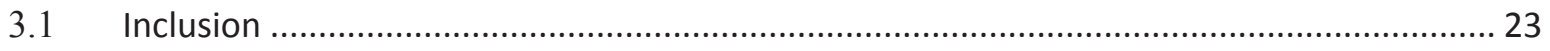

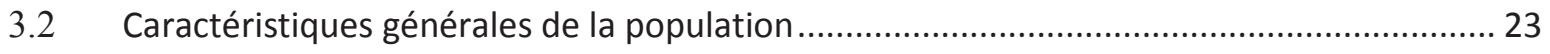

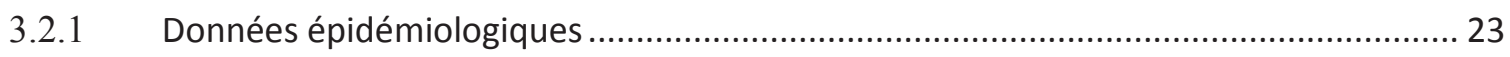

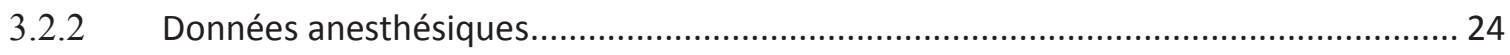

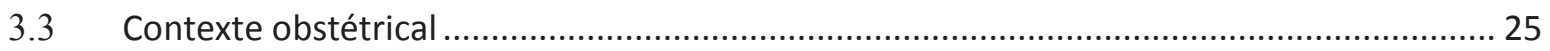

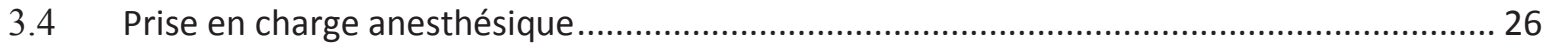

3.4.1 Prise en charge des voies aériennes supérieures ............................................. 26

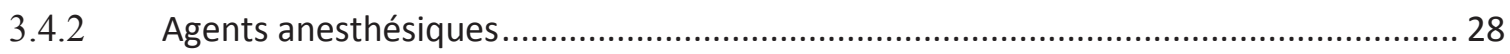

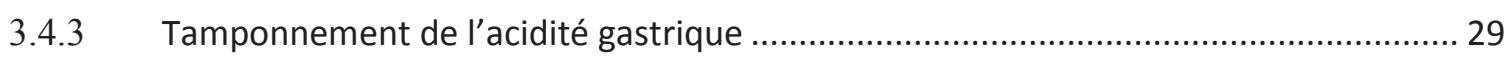

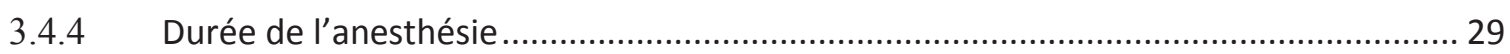

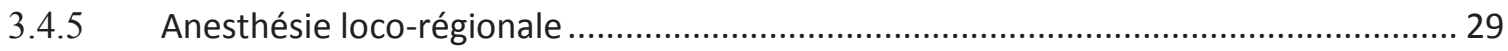

Facteurs influençant le choix de la technique anesthésique ........................................ 30

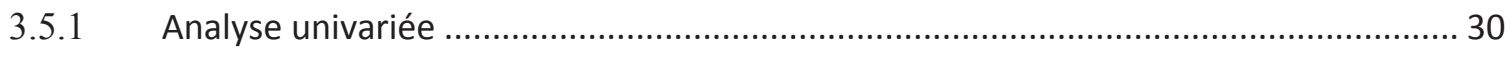

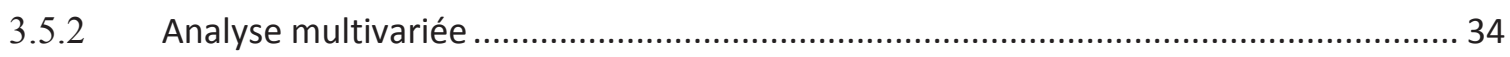

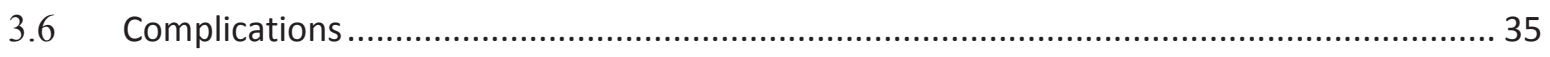

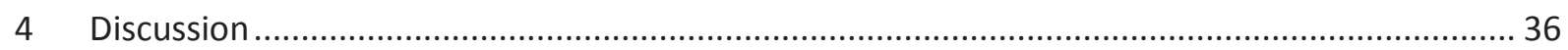

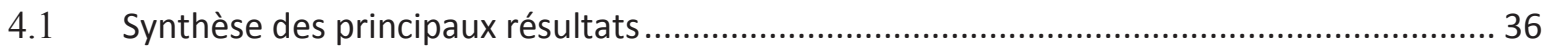

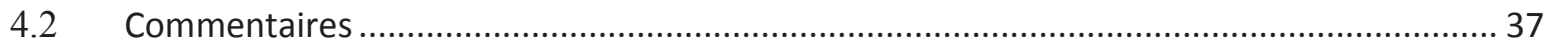

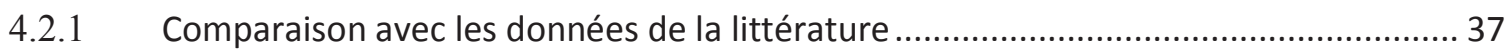

Intubation : la réticence des anesthésistes ....................................................... 38

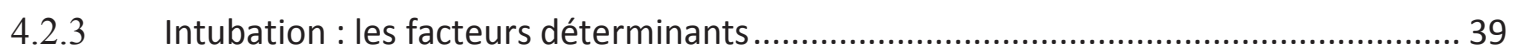

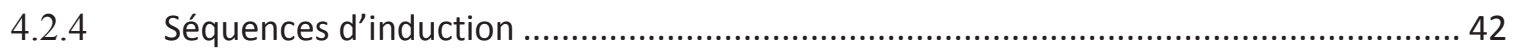

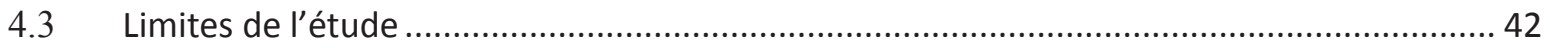

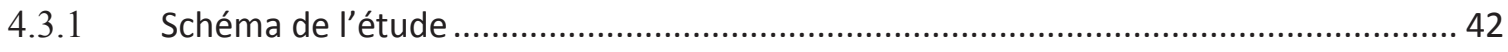




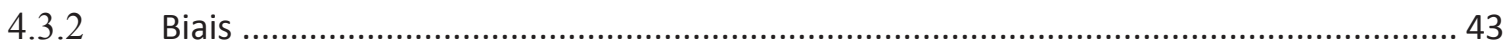

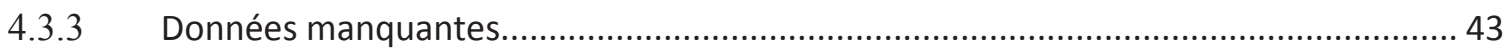

Place de l'anesthésie loco-régionale .................................................................. 44

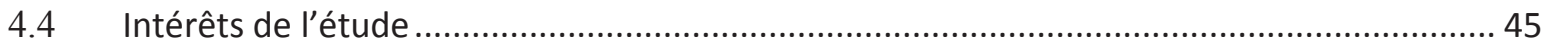

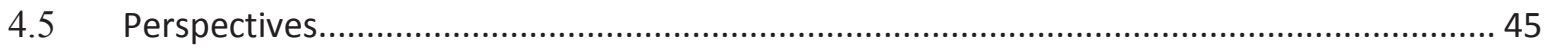

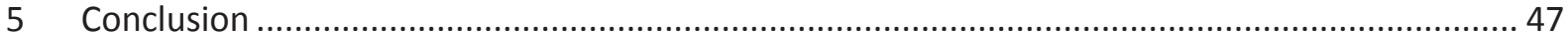

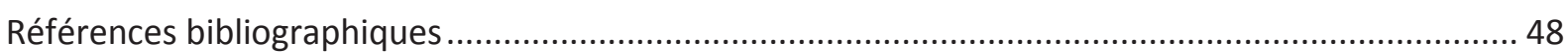




\section{Abréviations}

ALR : Anesthésie Loco-Régionale

Anti-H2 : Anti-histaminique $\mathrm{H} 2$

ASA : American Society of Anesthesiologists

ATCD : Antécédent

$\mathrm{CH}$ : Centre Hospitalier

CHU : Centre Hospitalier Universitaire

CNEMM : Comité National d’Experts sur la Mortalité Maternelle

CPP : Comité de Protection des Personnes

CRF : Capacité Résiduelle Fonctionnelle

DA : Délivrance Artificielle

DS : Déviation Standard

HPP : Hémorragie du Post-Partum

IMC : Indice de Masse Corporelle

IOT : Intubation Oro-Trachéale

RGO : Reflux Gastro-Oesophagien

RU : Révision Utérine

SA : Semaines d'Aménorrhée

SCA : Société Canadienne des Anesthésiologistes

SDRA : Syndrome de Détresse Respiratoire Aiguë

SFAR : Société Française d'Anesthésie et de Réanimation

VS : Ventilation Spontanée 


\section{Introduction}

La gestion de l'anesthésie générale en fin de travail, et plus particulièrement des voies aériennes supérieures, ne fait l'objet d'aucune recommandation spécifique alors que cette problématique concerne régulièrement les médecins anesthésistes ${ }^{1}$. Certaines particularités physiologiques de la femme enceinte incitent à favoriser l'induction en séquence rapide avec intubation oro-trachéale (IOT), mais le contexte d'urgence et d'intubation potentiellement difficile peuvent au contraire amener à privilégier une anesthésie en ventilation spontanée ${ }^{2}$. Ainsi, dans la littérature le taux d'IOT n'excède pas $20 \%$ dans ce contexte.

En l'absence de réponse claire de la part des sociétés savantes, de nombreuses questions restent en suspens quant à la technique anesthésique la plus appropriée : le rapport bénéfice-risque entre l'induction en séquence rapide et une anesthésie générale en ventilation spontanée n'est pas formellement établi et il n'existe actuellement pas de critère clinique permettant de choisir une technique plutôt qu'une autre. On observe donc dans la littérature de grandes disparités de pratiques selon les équipes.

En conséquence, l'objet de ce travail était d'évaluer les pratiques concernant la prise en charge des voies aériennes en fin de travail, au sein de trois centres hospitaliers de la région Haute-Normandie.

\subsection{Modifications anatomiques et physiologiques liées à la grossesse}

\subsubsection{Modifications des grandes fonctions vitales ${ }^{1,3}$}

\subsubsection{Modifications du système cardio-vasculaire}

Sous l'influence de l'imprégnation œstrogénique, le débit cardiaque augmente de $40 \%$ à $50 \%$ environ, par le biais de l'augmentation de l'inotropisme, du volume d'éjection systolique (de l'ordre de 10 à $15 \mathrm{ml}$ ) et de la fréquence cardiaque (de 10 à 15 battements par minute).

Il existe par ailleurs une vasodilatation artérielle périphérique due à la progestérone, entraînant une diminution de la pression artérielle alors même que la volémie est augmentée. Au niveau du système veineux, en fin de grossesse, la compression de la veine cave inférieure par l'utérus gravide provoque une diminution du retour veineux, pouvant avoir un retentissement hémodynamique majeur chez la mère et donc chez le fœtus. Ce phénomène, très dépendant de la position de la mère, est notamment majoré en décubitus dorsal. 
La volémie maternelle augmente tout au long de la grossesse pour atteindre à terme une majoration de $35 \%$ à $45 \%$ (soit 1 à 1,5l). Le volume plasmatique augmente plus que le volume globulaire, provoquant une hémodilution à l'origine de l'anémie physiologique de la grossesse. Cette rétention hydro-sodée est également due à l'augmentation du taux d'œstrogènes, ainsi qu'à l'activation du système rénine-angiotensine-aldostérone.

\subsubsection{Modifications du système respiratoire}

Au niveau pharyngo-laryngé, il existe un important œdème des muqueuses, en lien avec la rétention hydro-sodée précédemment décrite accompagnée d'une hyperhémie. Ceci entraîne une diminution du diamètre de la filière laryngée et un risque accru de saignement lors de l'intubation, rendant celleci plus difficile que dans la population générale ${ }^{4}$.

De plus, les modifications anatomiques au cours de la grossesse ont des conséquences importantes sur le système respiratoire : les patientes développent un élargissement du thorax avec élévation des côtes inférieures et augmentation de l'angle sous-costal, une élévation du diaphragme ainsi qu'une augmentation du diamètre transversal des poumons.

Parallèlement à ces modifications anatomiques, la grossesse a des répercussions fonctionnelles sur la respiration. Elle est marquée par une augmentation des besoins en oxygène, pour le fœtus et le placenta. Cela se traduit par une augmentation de la ventilation minute, secondaire à une majoration du volume courant (d'environ 45\%), la fréquence respiratoire étant quant à elle peu modifiée.

Cette augmentation du volume courant se fait aux dépens du volume de réserve expiratoire, qui chute de $20 \%$. Le volume résiduel chute également de $20 \%$, ce qui aboutit à une diminution de la capacité résiduelle fonctionnelle (CRF) de l'ordre de $40 \%$. La capacité pulmonaire totale ne varie pas. La conséquence principale de ces modifications est la diminution des réserves en oxygène et donc du temps d'apnée sans désaturation.

\subsubsection{Modifications du système digestif}

Du fait de l'imprégnation hormonale en progestérone il existe une baisse du tonus du sphincter inférieur de l'œsophage et donc une augmentation de la fréquence du reflux gastro-oesophagien ${ }^{5}$. Le volume gastrique et son acidité augmentent progressivement au cours de la grossesse.

Concernant la vidange gastrique, des données contradictoires sont retrouvées dans la littérature, mais il semblerait que celle-ci ne soit pas retardée au cours de la grossesse ${ }^{6}$. En revanche, au cours 
du travail obstétrical, il existe de façon certaine un ralentissement de la vidange gastrique (notamment en raison de l'administration fréquente de morphiniques pendant le travail) ${ }^{7}$.

C'est donc sur la base de ces données physiologiques qu'il est admis que la femme enceinte, dès 14 semaines d'aménorrhée $(S A)$, doit être considérée comme à " estomac plein » et donc à haut risque d'inhalation, et ce jusqu'à $48 \mathrm{~h}$ après l'accouchement (les modifications hormonales persistant en post-partum immédiat) ${ }^{8,9}$.

\subsubsection{Modifications liées à certains terrains ou états pathologiques}

\subsubsection{Obésité}

Lors d'une grossesse, la prise de poids moyenne est estimée à environ $12,9 \mathrm{~kg}$, en France, en $2003^{10}$. II n'est pas rare qu'une obésité vienne compliquer le terrain de la femme enceinte, d'autant plus que l'incidence de cette pathologie est en augmentation constante : en 2003, 22,6\% des femmes à terme avaient un IMC supérieur à $25 \mathrm{~kg} / \mathrm{m}^{2}$ contre 19,9\% en 1995.

L'existence d'une obésité a un retentissement direct sur la prise en charge des voies aériennes supérieures.

Tout d'abord, l'obésité est un facteur de risque indépendant de ventilation difficile. Même si cela est discuté dans la littérature, l'obésité semble également être un facteur de risque d'intubation difficile, que ce soit dans la population générale ou chez la femme enceinte ${ }^{11,12}$. Ces difficultés d'intubation peuvent provenir de l'augmentation du volume des seins, de la graisse de la face et des joues, d'un cou court, d'une grosse langue, d'une infiltration du palais et de la muqueuse pharyngée, d'un larynx haut situé, d'une limitation de l'ouverture de bouche et d'une limitation de la mobilité du rachis cervical.

De plus, l'obésité est à l'origine d'un syndrome respiratoire restrictif associé à une diminution de la CRF, déjà diminuée par la grossesse elle-même, ce qui a pour conséquence une diminution du temps d'apnée sans désaturation.

L'augmentation du risque de difficultés techniques chez les patientes obèses et la mauvaise tolérance à l'apnée, au cas où ces difficultés se présentent, augmentent donc les risques liés à l'intubation.

\subsubsection{Pré-éclampsie}

La pré-éclampsie est une pathologie maternelle exclusivement liée à la grossesse et pouvant avoir un retentissement direct sur la gestion des voies aériennes supérieures. Cette pathologie est définie par 
une hypertension artérielle gravidique associée à une protéinurie supérieure à $0,3 \mathrm{~g} / 24 \mathrm{~h}$. Cette protéinurie est à l'origine d'une chute de la pression oncotique responsable de la présence d'importants œdèmes. Ces œdèmes sont notamment marqués au niveau du visage, du cou et des structures oro-pharyngées, diminuant ainsi le diamètre des voies aériennes supérieures ${ }^{13}$. Ils peuvent apparaître et se majorer très rapidement. De plus, il existe une hyperhémie qui, associée aux troubles de l'hémostase existants dans la pré-éclampsie, peut être à l'origine de saignements importants en cas d'abord difficile des voies aériennes supérieures. Ainsi dans la conférence d'experts sur l'intubation difficile révisée en 2006, la Société Française d'Anesthésie et de Réanimation (SFAR) considère la pré-éclampsie comme une situation clinique à risque d'intubation difficile $^{14}$. Par ailleurs, il est important de noter qu'il existe également un risque d'obstruction laryngée à l'extubation.

\subsection{Morbi-mortalité maternelle en France}

\subsubsection{Généralités}

La mortalité maternelle est définie par " le décès d'une femme survenu au cours de la grossesse ou dans un délai de 42 jours après sa terminaison, quelle qu'en soit la durée ou la localisation, pour une cause quelconque déterminée ou aggravée par la grossesse ou les soins qu'elle a motivés, mais ni accidentelle, ni fortuite ».

Depuis 1996, il existe en France un système de surveillance de la mortalité maternelle. Le rapport du Comité National d'Experts sur la Mortalité Maternelle (CNEMM) de 2001 à 2006, paru en 2010 dans le Bulletin Epidémiologique Hebdomadaire, mettait en évidence un taux de décès estimé entre 9 et 13 pour 100000 naissances vivantes, ce qui correspond à environ 70 femmes par $a^{15}$.

Une première partie de ce rapport a permis d'établir les principales causes de décès maternel ${ }^{16}$ : les hémorragies (25\%), les complications de l'hypertension artérielle (12,3\%), les maladies thromboemboliques $(9,9 \%)$, les embolies amniotiques, les infections, les complications obstétricales indirectes, les cardiomyopathies du post-partum et enfin, les complications anesthésiques.

Dans le dernier rapport du CNEMM de 2007 à 2009, paru en novembre 2013, le taux de décès maternel était de 10,3 pour 100000 naissances vivantes ${ }^{17}$. 


\subsubsection{La mortalité maternelle liée à l'anesthésie}

En France, en 1999, la mortalité entièrement ou partiellement attribuable à l'anesthésie était respectivement de 0,69 pour 100000 anesthésies et de 4,7 pour 100000 anesthésies (toutes spécialités confondues) $)^{18}$.

D’après le rapport du CNEMM de 2001 à 2006, les décès maternels liés à l'anesthésie étaient au nombre de 7 entre 2001 et 2006, soit un taux global de mortalité de 0,14/100000 naissances vivantes (entre 1996 et 1998, six décès avaient eu lieu et un seul sur la période 1999-2001).

Parmi les 7 décès attribués aux complications de l'anesthésie entre 2001 et 2006, 5 ont pu être analysés par le comité d'expert. Tout d'abord, il est important de noter que tous les décès étaient survenus dans les suites d’une anesthésie générale. Trois des cinq décès étaient dus à un choc anaphylactique à la succinylcholine (documenté ou fortement suspecté), 1 décès était dû à un syndrome de Mendelson survenu secondairement en réanimation (patiente hospitalisée en réanimation pour une défaillance myocardique apparue au cours d'une césarienne) et le $5^{\text {ème }}$ décès a été attribué soit à une intubation œsophagienne, soit à un choc anaphylactique à la succinylcholine. Une autre étude, relatée dans ce même rapport, s'est intéressée plus spécifiquement à la mortalité maternelle directement liée aux césariennes entre 1996 et $2000^{19}$. Cette étude montrait que l'accouchement par césarienne était associé à un risque accru de décès maternel. Cette majoration du risque de décès en cas de césarienne pouvait être en partie attribuée aux complications de l'anesthésie générale puisque 3 des 4 décès étaient survenus lors d'une anesthésie générale alors que seules $12 \%$ des césariennes avaient été réalisées sous anesthésie générale, en France, au cours de l’année 1998.

Le dernier rapport du CNEMM de 2007 à 2009 fait état, pour sa part, de 3 décès liés aux complications de l'anesthésie, soit un taux de 0,1 pour 100000 naissances vivantes. Parmi ces 3 décès, un seul a pu être expertisé, et a été attribué à un choc anaphylactique à la succinylcholine ${ }^{17}$.

\subsubsection{Les principales complications de l'anesthésie générale en obstétrique}

\subsubsection{L'anaphylaxie}

En France, l'incidence des réactions anaphylactiques d'origine allergique est estimée à 100,6/million d'anesthésies, avec une nette prépondérance féminine puisque le sexe-ratio est de 55,4/154,9 $(0,35)^{20}$.

Les substances les plus fréquemment en cause sont les curares, suivis du latex, puis des antibiotiques. 
Le rapport du CNEMM de 2001 à 2006, avait identifié le choc anaphylactique comme étant une cause importante de mortalité maternelle : en effet, 3 (voire 4) des 7 décès survenus étaient liés à un choc anaphylactique $^{15}$. Le rapport du CNEMM de 2007 à 2009 allait également dans ce sens, puisque le seul des 3 décès expertisé était également dû à un choc anaphylactique ${ }^{17}$. Le produit en cause était dans tous les cas la succinylcholine. Or, la succinylcholine reste le curare de choix dans le cas de l'anesthésie générale avec induction en séquence rapide chez la femme enceinte, en raison du risque d'inhalation.

\subsubsection{Le syndrome de Mendelson}

Le syndrome d'inhalation bronchique du contenu gastrique au cours de l'anesthésie générale, autrement appelé syndrome de Mendelson, est l'une des complications les plus redoutées en anesthésie et une préoccupation majeure de l'anesthésiste. II avait été initialement décrit par Mendelson en 1946, chez la femme enceinte ${ }^{21}$.

La physiopathologie du syndrome d'inhalation de liquide gastrique repose sur deux mécanismes différents : la régurgitation (passive) ou le vomissement (actif), avec dans les deux cas un passage du contenu gastrique dans les bronches induisant des lésions pulmonaires de gravité variable: inhalation asymptomatique, pneumopathie d'inhalation, et au maximum le syndrome de détresse respiratoire aiguë de l'adulte (SDRA).

Les facteurs déterminants de la gravité de l'atteinte pulmonaire sont : un volume gastrique résiduel supérieur à $0,4 \mathrm{ml} / \mathrm{kg}$, un $\mathrm{pH}$ inférieur à 2,5 (la sévérité de l'agression pulmonaire est proportionnelle à l'acidité du $\mathrm{pH}$ ) et la présence ou non de particules alimentaires, responsables d'obstructions et d'atélectasies ${ }^{22}$.

Chez la personne consciente, il existe des moyens de protection physiologiques de l'arbre trachéobronchique en cas de régurgitation ou de vomissement. Ces moyens, constitués par la déglutition, la fermeture de la glotte et la toux, sont sous le contrôle du système nerveux central. Lors d'une anesthésie générale ces mécanismes sont altérés par l'effet dépresseur des agents anesthésiques sur le système nerveux central. De ce fait, les voies aériennes supérieures sont particulièrement exposées à la phase d'induction anesthésique (avant la mise en place de la sonde d'intubation trachéale qui permet la protection partielle des voies aériennes supérieures grâce au ballonnet intratrachéal) et à la phase de réveil (après extubation et avant une reprise totale de la conscience) ${ }^{23}$.

L'incidence du syndrome de Mendelson varie selon les études, mais reste relativement faible : de $1 / 2000$ à $1 / 8000$ anesthésies générales ${ }^{22,24-27}$. Cette incidence peut être augmentée dans certaines 
situations cliniques, notamment chez la femme enceinte (pour les raisons explicitées précédemment) et dans les situations d'urgence où la période de jeûne pré-anesthésique requise n'a pas été respectée.

La mortalité due à ce syndrome est de l'ordre de 1 pour 220000 anesthésies générales, soit un cinquième de l'ensemble des décès entièrement ou partiellement imputables à l'anesthésie générale ${ }^{28}$. Si l'on inclut uniquement les anesthésies générales avec intubation ou mise en place d'un masque laryngé, ce taux augmente à environ 1 pour 120000 anesthésies générales.

En obstétrique ce taux de mortalité est comparable. En France, dans le rapport du CNEMM de 2001 à $2006^{15}, 1$ des 7 décès était lié à un syndrome de Mendelson (survenu à distance de l'accouchement, au cours d'une réintubation chez une patiente hospitalisée en réanimation pour insuffisance cardiaque gauche sévère, apparue dans les suites d'une hémorragie du post-partum). En Angleterre, 1 des 6 décès survenus sur la période 2000-2002 était également lié à ce syndrome, apparu au décours d'un échec d'intubation oro-trachéale ${ }^{29}$.

Afin de limiter les risques d'inhalation, plusieurs moyens peuvent être mis en œuvre dans les situations à risque. En premier lieu, dès que cela est possible, il convient de favoriser l'anesthésie loco-régionale (ALR) par rapport à l'anesthésie générale. En effet, seuls des cas anecdotiques d'inhalation ont été décrits chez des patients ayant bénéficié d'une ALR, rapportés à une prémédication trop importante entraînant une altération de la protection des voies aériennes supérieures.

Lorsque l'anesthésie générale s'impose, par analogie avec les recommandations pour la prise en charge anesthésique en cas d'estomac plein, une induction en séquence rapide avec intubation orotrachéale est préconisée ${ }^{30}$.

\subsubsection{L'intubation difficile/impossible}

La Société Française d'Anesthésie et de Réanimation définit l'intubation difficile par la nécessité de plus de deux laryngoscopies et/ou la mise en œuvre d'une technique alternative après optimisation de la position de la tête, avec ou sans manipulation laryngée externe. Les critères d'intubation difficile déterminés par la SFAR seront détaillés dans le chapitre suivant.

L'incidence de l'intubation impossible en obstétrique est habituellement considérée comme étant 8 fois supérieure à celle de l'intubation impossible dans la population générale ${ }^{31-33}$, soit 1/280 à 1/250 contre $1 / 3000$ dans la population générale. L'incidence de l'intubation difficile est de $1 / 30^{34}$ en 
obstétrique, contre environ $1 \%$ dans la population générale. Cependant une récente revue de la littérature n'a pas mis en évidence de différence statistiquement significative entre le taux d'intubation difficile en obstétrique et dans la population générale, remettant ainsi en question la notion habituellement retenue que l'intubation est plus difficile chez les parturientes que dans la population générale $e^{32}$.

En France, parmi les décès analysés par le CNEMM de 2001 à 2006, aucun n'était directement lié à une intubation difficile, mais 1 des 7 décès était possiblement attribuable à une intubation œsophagienne, ce qui peut laisser penser que des difficultés d'intubation s'étaient présentées.

En Angleterre, une récente étude réalisée à partir des données de I'UK Obstetric Surveillance System de 2008 à 2010, montrait également l'absence de décès maternels dus à une difficulté de prise en charge des voies aériennes supérieures ${ }^{31}$. Cependant, sur la période 2000-2002, 3 décès étaient survenus dans ce contexte ${ }^{29}$.

Afin d'anticiper au mieux la survenue d'une intubation difficile/impossible, une évaluation systématique des voies aériennes supérieures doit avoir lieu en consultation d'anesthésie et doit idéalement être répétée en consultation pré-anesthésique.

\subsection{Evaluation des voies aériennes supérieures en obstétrique}

\subsubsection{Généralités}

Avant la réalisation de tout acte anesthésique, le praticien se doit d'évaluer l'accès aux voies aériennes supérieures, afin d'anticiper au mieux une éventuelle intubation difficile. Pour cela différents facteurs de risque cliniques sont à rechercher et différents scores d'évaluation existent, mais aucun n'est spécifique à la femme enceinte.

La SFAR a défini plusieurs critères prédictifs d'une intubation difficile à rechercher ${ }^{14}$ : un antécédent d'intubation difficile, une classe de Mallampati supérieure à $2 \mathrm{~cm}$, une distance thyromentonnière inférieure à $6 \mathrm{~cm}$ et une ouverture de bouche inférieure à $35 \mathrm{~mm}$. II est également conseillé d'évaluer la mobilité mandibulaire (par le test de morsure de langue) et la mobilité du rachis cervical (flexion et extension). Enfin, certaines situations cliniques sont considérées comme à risque d'intubation difficile, notamment l'obésité avec un IMC $>35 \mathrm{~kg} / \mathrm{m}^{2}$, ou la pré-éclampsie.

Les facteurs spécifiques associés à l'intubation difficile en obstétrique ont été étudiés par Rocke et al. en $1992^{35}$. Ceux-ci étaient : une classe de Mallampati 2, 3 ou 4, un cou court, un rétrognathisme, et des incisives proéminentes. 


\subsubsection{Particularités de l'évaluation des voies aériennes supérieures en obstétrique}

En ce qui concerne l'évaluation des voies aériennes supérieures, une des spécificités de l'obstétrique est l'évolution de la classe de Mallampati au cours de la grossesse et au cours du travail.

La classe de Mallampati est évaluée chez un sujet éveillé, assis ou debout, qui ouvre la bouche aussi grand que possible et tire la langue aussi loin que possible, en l'absence de phonation ${ }^{36}$. La classification est établie en fonction des structures oro-pharyngées visualisées.

Les 4 classes de Mallampati sont :

- Classe 1 : toute la luette et les loges amygdaliennes sont visibles

- Classe 2 : la luette est partiellement visible

- Classe 3 : le palais membraneux est visible

- Classe 4 : seul le palais osseux est visible

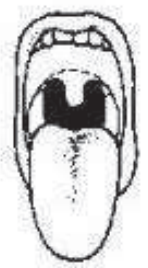

Cass 1

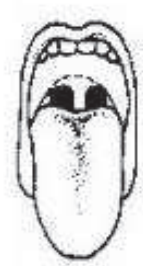

Cass 2

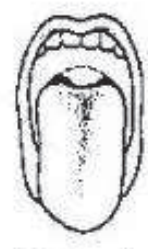

Cass 3

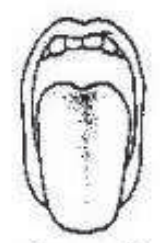

Cass 4

Les classes 3 et 4 sont prédictives d'une intubation difficile.

L'utilisation de cette classification en obstétrique a été validée par l'étude de Rocke et al. ${ }^{35}$ qui a montré une bonne corrélation avec l'intubation difficile, chez 1500 parturientes intubées pour une césarienne programmée ou en urgence. Dans cette étude, le risque relatif d'une intubation difficile était de 3,2 pour une classe 2 de Mallampati, 7,6 pour une classe 3 et 11,3 pour une classe 4 . D’autres potentiels critères prédictifs d'intubation difficile avaient été évalués (l'obésité, le cou court, la dentition maxillaire, le rétrognathisme mandibulaire, l'œdème de la face et de la langue), mais la classe de Mallampati restait le facteur le plus fortement corrélé à la difficulté de l'intubation trachéale.

Par ailleurs, plusieurs études ont décrit une évolution de la classe de Mallampati au cours de la grossesse et au cours du travail. Dans une première étude, la classe de Mallampati était évaluée à 12 SA puis à 38 SA chez 242 parturientes ${ }^{37}$. L'incidence de la classe 4 augmentait de $34 \%$ entre les deux périodes, et cette aggravation était corrélée à la prise de poids. Une étude plus récente s'est intéressée à l'évolution de la classe de Mallampati au cours du travail obstétrical chez 61 patientes ${ }^{38}$. La classe de Mallampati était déterminée en début de travail, 20 minutes après la délivrance, puis à 
36-48h de post-partum. Les résultats montrent une évolution significative des classes de Mallampati entre le début et la fin du travail : augmentation d'une classe pour 20 parturientes (33\%) et de deux classes pour 3 parturientes (5\%). A la fin du travail, 8 parturientes avaient une classe 4 (la présence initiale d'une classe 4 était un critère d'exclusion) et 30 femmes (50 \%) avaient une classe Mallampati 3 ou $4(p<0,01)$. Chez certaines patientes, le changement de classe par rapport à l'évaluation initiale persistait à 36-48h. II n'y avait pas d'association entre ces changements de classe et le poids - ou la taille - des parturientes, la durée du travail ou la quantité d’apports liquidiens.

Ces constatations soulignent l'importance d'une évaluation répétée des voies aériennes supérieures dans le contexte de l'anesthésie obstétricale. Une première évaluation est faite au moment de la consultation d'anesthésie (qui a généralement lieu au $7^{\text {ème }}$ mois), mais il paraît indispensable de réévaluer le risque d'intubation difficile à l'arrivée des patientes en salle de naissance et avant toute induction anesthésique.

\subsection{Anesthésie générale en fin de travail}

\subsubsection{Evolution des pratiques en obstétrique et en anesthésie obstétricale}

Sur les dix dernières années, on observe une évolution des pratiques obstétricales ainsi que des pratiques en anesthésie obstétricale : en effet, l'enquête nationale périnatale 2010 a montré une légère augmentation du taux de césarienne entre 1998 et 2010 (de 17,5 à 21\%), mais sans augmentation du taux de césarienne au cours du travail ${ }^{39}$. On observe également une augmentation du nombre d'anesthésies péridurales réalisées (de 62,6 à 70\%), entraînant la diminution du nombre d'anesthésies générales pratiquées au cours du travail (pour césarienne ou autre) qui passe de 2,6 à 1,2\% entre 1998 et 2010. En cas de voie basse instrumentale, ce taux était de 0,6\% en 2010.

Cette évolution des pratiques s'explique très probablement par le fait que le risque anesthésique est moindre avec l'ALR qu'avec l'anesthésie générale, incitant l'anesthésiste à privilégier l'ALR ${ }^{29,40}$. Les indications de l'anesthésie générale se réduisent donc aux situations où l'ALR est contre-indiquée et aux situations d'urgence où l'extraction fœtale doit être la plus rapide possible, ne permettant pas le délai nécessaire à la réalisation de l'ALR.

Une des conséquences directes de la raréfaction des indications de l'anesthésie générale en obstétrique est la diminution de l'expérience des praticiens dans ce domaine et donc un apprentissage devenu plus difficile pour les plus jeunes.

A la problématique de la difficulté de la gestion des voies aériennes en fin de travail s'ajoute donc celle du manque d'expérience et de formation sur la gestion de ces situations particulièrement urgentes et à risque pour la mère et l'enfant. 


\subsubsection{Indications de l’anesthésie générale en salle de naissance}

Actuellement le taux d'anesthésies générales réalisées en salle de naissance est de l'ordre de $1 \%^{39}$. Le pré-requis à la réalisation d'une anesthésie générale en salle de naissance est l'absence, l'inefficacité, ou la contre-indication de l'anesthésie péri-médullaire. Cette contre-indication peut être liée à la patiente elle-même (refus de la patiente, troubles de la coagulation, infection maternelle, infection au point de ponction...) ou à la situation obstétricale (extraction fœtale urgente, hémorragie de la délivrance avec instabilité hémodynamique...).

Dans ce cas, une anesthésie générale est nécessaire pour les gestes suivants :

- La révision utérine associée ou non à la délivrance artificielle (fréquent contexte d'hémorragie de la délivrance)

- L'extraction fœtale, manuelle (type version-grande extraction) ou instrumentale

- La suture d'une épisiotomie ou d'une déchirure périnéale (lorsque l'anesthésie locale ne suffit pas)

L'anesthésie générale en salle de naissance concerne donc des gestes réalisés en fin de travail, ou en post-partum immédiat.

Dans ces indications, l'anesthésie générale est fréquemment réalisée dans un contexte d'urgence (urgence obstétricale, ou maternelle, par exemple en cas d'hémorragie du post-partum sévère).

\subsubsection{Prévention du syndrome de Mendelson}

\subsubsection{Règles de jeûne au cours du travail obstétrical}

Le ralentissement de la vidange gastrique au cours du travail et l'administration fréquente d'opioïdes, favorisant eux-mêmes ce retard, ont conduit les sociétés savantes à établir des recommandations sur la question du jeûne au cours du travail obstétrical.

Pour rappel, la période de jeûne pré-opératoire recommandée avant toute chirurgie est de 6 heures pour les solides et de 2 heures pour les liquides clairs ${ }^{41}$.

L'American Society of Anesthesiologists (ASA) recommande l'absence totale de prise de solides au cours du travail obstétrical ${ }^{42}$. Elle autorise la prise de petites quantités de liquides clairs (eau, jus de fruits sans pulpe, thé, café, boissons sucrées non gazeuses) précisant que les patientes les plus à risque d'inhalation (obèses, diabétiques...) doivent faire l'objet de restrictions plus importantes, à évaluer au cas par cas. 
La Société Canadienne des Anesthésiologistes (SCA) va dans le même sens, puisque dans son guide d'exercice de l'anesthésie 2013, elle précise que «les parturientes ne devraient pas absorber d'aliments solides une fois le travail actif débuté ${ }^{43}$. L'absorption de liquides clairs est en revanche autorisée.

La SFAR quant à elle, se prononce de façon moins ferme en stipulant que «le jeûne de la femme enceinte est remis en question. La femme en travail bénéficiant d'une analgésie périmédullaire peut être autorisée à absorber des liquides non particulaires (grade B), sauf en cas de diabète, d'obésité morbide ou de césarienne prévisible ${ }^{44}$. II n'est pas fait état du jeûne chez les patientes n'ayant pas bénéficié d'une anesthésie périmédullaire, mais on peut penser que celles-ci étant plus exposées au risque de nécessiter une anesthésie générale, le principe du jeûne strict devrait s'appliquer d'autant plus.

Ces recommandations ont été faites alors même que le jeûne au cours du travail est actuellement remis en question ${ }^{45}$. Le confort des patientes mis en balance avec la rareté du syndrome d'inhalation est avancé comme argument contre le jeûne strict au cours du travail. De plus, le véritable effort que représentent le travail et l'accouchement pourrait également justifier l'intérêt des apports caloriques au cours du travail. Cependant, en raison du ralentissement de la vidange gastrique au cours du travail ${ }^{7}$ et compte tenu des études ayant mis en évidence un risque plus important de vomissements après ingestion de solides ${ }^{46}$, le principe de précaution a été appliqué, aboutissant aux recommandations actuelles.

\subsubsection{Tamponnement de l'acidité gastrique}

La prévention médicamenteuse du syndrome de Mendelson est recommandée en obstétrique avant tout acte chirurgical sous anesthésie générale. De façon plus générale, cette prophylaxie est indiquée avant toute anesthésie générale chez les patients à risque d'inhalation ${ }^{30}$.

Différentes classes médicamenteuses peuvent être utilisées: les antiacides non particulaires, les inhibiteurs de la pompe à protons, les antihistaminiques $\mathrm{H} 2$ (anti-H2) et les prokinétiques.

Une récente revue de la littérature ${ }^{47}$ a mis en évidence une supériorité des anti-H2 combinés aux antiacides, sous forme effervescente. L'efficacité des anti-H2 est due à l'élévation du pH gastrique et à la diminution du volume gastrique (par blocage de la sécrétion acide de l'estomac), et la combinaison avec les antiacides permet une efficacité immédiate et prolongée. 


\subsubsection{L'induction en séquence rapide}

Comme il a été vu précédemment, la meilleure façon de prévenir le syndrome de Mendelson chez les patients à risque est d'avoir recours à l'anesthésie loco-régionale et d'éviter ainsi l'anesthésie générale, bien plus pourvoyeuse d'inhalation.

Mais lorsque l'anesthésie générale s'impose chez une patiente enceinte (et donc à risque d'inhalation), il est classique de considérer qu'une induction en séquence rapide doit être réalisée ${ }^{30}$. Elle permet d'assurer la protection des voies aériennes supérieures (via la sonde d'intubation) plus rapidement que lors d'une technique d'induction classique, et limite les risques de vomissement en évitant l'utilisation d'un morphinique. Cette induction en séquence rapide comporte plusieurs étapes, parfaitement standardisées :

- Pré-oxygénation

- Manœuvre de Sellick (pression cricoïdienne)

- Administration d'un hypnotique intra-veineux, au choix: le thiopental, le propofol, l'étomidate ou la kétamine

- Administration d'un curare intra-veineux, le curare de choix étant la succinylcholine

- Laryngoscopie et intubation oro-trachéale

\subsubsection{Anesthésie générale en salle de naissance et gestion des voies aériennes}

\subsubsection{Les techniques anesthésiques}

L'induction en séquence rapide est la technique de choix lorsque l'on veut privilégier la prévention du risque d'inhalation.

Mais il existe d'autres techniques alternatives, permettant notamment de s'affranchir du risque d'intubation difficile, voire impossible, en conservant une ventilation spontanée, et de limiter le risque anaphylactique en évitant l'utilisation de curare.

Les gestes obstétricaux réalisés en fin de travail sont souvent des gestes peu invasifs et de relativement courte durée, pouvant permettre une anesthésie générale en ventilation spontanée ${ }^{48,49}$. Les drogues utilisées devront permettre le maintien du contrôle central de la ventilation et une moindre dépression des muscles respiratoires. Pour cela, les drogues de choix sont : la kétamine, le propofol, le rémifentanil, l'alfentanil et le midazolam ${ }^{49}$. Elles peuvent être administrées seules ou en association mais toujours à faible dose et en titration, de manière à conserver une ventilation spontanée. Celle-ci se fera dans la majorité des cas au masque facial, en s'assurant du maintien de la perméabilité de la filière aérienne par la position de la tête en hyper-extension associée à la sub- 
luxation antérieure de la mandibule. La problématique principale en cas d'anesthésie générale en ventilation spontanée est l'absence de protection des voies aériennes supérieures, puisque l'anesthésie générale entraîne une perte des réflexes de protection des voies aériennes supérieures et que l'étanchéité n'est pas assurée (puisqu'il n'y a pas de sonde d'intubation oro-trachéale). Parmi les agents anesthésiques couramment utilisés pour ce type de procédure, la kétamine offre l'avantage d'un maintien relatif des réflexes de protection des voies aériennes supérieures ${ }^{50}$.

Les techniques d'anesthésie générale en ventilation spontanée avec aide inspiratoire et/ou avec utilisation d'agents halogénés ne seront pas décrites, puisque l'équipement des salles de naissance ne permet pas d'avoir recours à ces techniques.

\subsubsection{Ce qu'en disent les sociétés savantes}

En France, il n'existe, à ce jour, pas de recommandation spécifique concernant la gestion des voies aériennes supérieures dans l'anesthésie générale en fin de travail, en salle de naissance.

Dans la conférence d'actualisation de la SFAR en 2000 sur l'urgence anesthésique obstétricale, la seule référence à l'anesthésie générale en fin de travail concerne la révision utérine pour délivrance artificielle $^{51}$. II en ressort qu'en l'absence d'anesthésie péridurale, il convient de réaliser une anesthésie générale avec les mêmes précautions que celles appliquées dans l'anesthésie générale pour césarienne, à savoir une induction en séquence rapide. On peut probablement extrapoler cette recommandation à toute anesthésie générale réalisée en fin de travail ou en post-partum immédiat, mais il n'existe pas de recommandation spécifique clairement établie.

De plus, dans la conférence d'actualisation sur l'anesthésie pour estomac plein, revue en $2005^{30}$, il est bien précisé que la femme enceinte est à risque d'inhalation dès 14 semaines d'aménorrhée. On en déduit donc que l'anesthésie générale devrait être réalisée avec une induction en séquence rapide. Mais là encore, la question de la période de fin de travail et du post-partum immédiat, n'est pas précisément abordée.

Dans ses recommandations pour la pratique clinique en anesthésie obstétricale l'ASA a, quant à elle, émis des recommandations concernant l'anesthésie pour délivrance artificielle ${ }^{42}$. Celles-ci stipulent qu'en cas d'absence ou de contre-indication à une anesthésie péri-médullaire (qui reste la technique de choix), « la prévention de l'inhalation devrait être prise en considération. La sédation/analgésie devrait être titrée prudemment en raison du risque de dépression respiratoire et d'inhalation en post-partum immédiat ». En cas d’hémorragie de la délivrance sévère, " une anesthésie générale avec intubation oro-trachéale serait préférable à une anesthésie péri-médullaire ». 
On constate donc qu'en dehors du cas de l'hémorragie de la délivrance sévère, les recommandations restent assez évasives concernant la gestion des voies aériennes supérieures dans l'anesthésie générale en salle de naissance, bien que le risque d'inhalation soit souligné.

\subsubsection{Ce qui est fait en pratique}

D’après les données exposées précédemment, on comprend bien que l'anesthésiste est confronté à une double problématique lorsqu'il s'agit de réaliser une anesthésie générale en fin de travail ou en post-partum immédiat :

- D'une part le risque d'inhalation chez la femme enceinte, qui est plutôt en faveur d'une anesthésie générale avec induction en séquence rapide, selon le respect des bonnes pratiques

- Mais d'autre part le risque de complications liées à cette même induction en séquence rapide, telles que l'intubation difficile/impossible, particulièrement redoutées chez la femme enceinte, ou l'anaphylaxie, incitant plutôt à réaliser une anesthésie générale en ventilation spontanée

Le choix d'une technique qui s'imposerait est d'autant plus difficile que, comme nous l'avons constaté en analysant les décès liés à l'anesthésie, l'induction en séquence rapide ne prémunit pas totalement contre l'inhalation en cas de difficulté d'intubation.

C'est donc après analyse de cette balance risque/risque que le praticien va choisir la technique lui semblant la plus appropriée.

Actuellement, le taux d'intubation dans ce contexte se situe entre 5 et $20 \%$ environ ${ }^{48,52-54}$. 
Il existe donc actuellement une discordance majeure entre ce qui semblerait être, au vue des données physiopathologiques, la technique anesthésique la plus sécuritaire pour contrer le risque d'inhalation (à savoir l'intubation en séquence rapide), et ce qui est fait concrètement sur le terrain, par les praticiens. En effet, en privilégiant l'anesthésie générale en ventilation spontanée, ceux-ci semblent prendre en compte, en priorité, les risques d'anaphylaxie et de difficultés d'intubation et/ou de ventilation, inhérents à l'intubation en séquence rapide.

Cet écart entre le principe de l'intubation oro-trachéale systématique chez la femme enceinte et la réalité pratique, ainsi que les interrogations qu'il suscite, nous ont conduits à mener ce travail qui avait pour objectif principal d'évaluer les pratiques dans cette situation particulière qu'est l'anesthésie générale en fin de travail. Nous avons également cherché à comprendre quelles pouvaient être les raisons de cet écart, et quelles en étaient les éventuelles conséquences. 


\section{Matériel et méthodes}

\subsection{Caractéristiques générales de l'étude}

II s'agissait d'une étude observationnelle, rétrospective, multicentrique, réalisée sur deux ans, de janvier 2009 à décembre 2010.

Trois centres ont participé à l'étude, tous situés en région Haute-Normandie :

- Le centre hospitalier universitaire de Rouen (hôpital Charles Nicolle) qui comprend une maternité de niveau III dans laquelle ont lieu environ 2800 accouchements par an.

- Le centre hospitalier du Belvédère (situé à Mont Saint-Aignan) qui comprend une maternité de niveau II dans laquelle ont lieu environ 3600 accouchements par an.

- Le centre hospitalier Les Feugrais (situé à Elbeuf) qui comprend une maternité de niveau II, dans laquelle ont lieu environ 1800 accouchements par an.

\subsection{Objectifs de l'étude et critères de jugement}

\subsubsection{Objectifs de l'étude}

L'objectif principal de l'étude était d'évaluer les pratiques concernant la prise en charge des voies aériennes dans les anesthésies générales réalisées en fin de travail, en salle de naissance.

Les objectifs secondaires étaient :

- Rechercher la présence éventuelle de facteurs influençant le choix de la technique anesthésique.

- Evaluer les complications survenues au décours de la prise en charge anesthésique.

\subsubsection{Critères de jugement}

Le critère de jugement principal de l'étude était le taux d'intubation oro-trachéale.

Les autres critères étudiés étaient : le taux de complication et les facteurs influençant le choix de la technique anesthésique (liés au contexte clinique ou au terrain des patientes). 


\subsection{Critères d'inclusion et d'exclusion}

Les critères d'inclusion étaient :

- Accouchement par voie basse, dans l'un des trois centres concernés, entre janvier 2009 et décembre 2010

- Anesthésie générale pour manœuvre obstétricale en fin de travail ou en post-partum immédiat (extraction fœtale, révision utérine et/ou délivrance artificielle, révision sous valve, suture d'épisiotomie ou de déchirure périnéale), ayant ou non bénéficié au préalable d'une anesthésie péri-médullaire

II n'y avait pas de critère d'exclusion. Les patientes ayant accouché avant un terme compatible avec la vie (interruption médicale de grossesse, mort fœtale in utero) n'étaient pas exclues.

\subsection{Recueil des données}

\subsubsection{Inclusion des patientes}

La liste des patientes à inclure a été obtenue à partir de différents moyens selon les centres.

Au CHU de Rouen, deux moyens ont été utilisés :

- D'une part, une demande a été faite au département d'information médicale, afin de retrouver à partir du logiciel de codage diagnostique la liste des patientes incluables. Les critères "anesthésie générale en salle de naissance ", "révision utérine, délivrance artificielle ou extraction fœtale instrumentale " ont été recherchés. A partir de la liste obtenue (152 patientes), tous les dossiers ont été étudiés, et seuls les dossiers correspondants réellement aux critères d'inclusion de l'étude ont été retenus (62 dossiers). La plupart des dossiers non retenus correspondaient à des cas où le geste obstétrical avait été réalisé sous péri-rachianesthésie (codage particulier), ou sous protoxyde d'azote, sans anesthésie péri-médullaire ni générale.

- D'autre part, le registre de salle de naissance a été étudié : il s'agit d'un registre présent en salle de naissance dans lequel tous les actes anesthésiques pratiqués en salle de naissance sont reportés. L'étude de ce registre a permis de faciliter le tri de la liste obtenue à partir du codage informatique et de récupérer huit dossiers n'ayant pas été sélectionnés informatiquement. Ceci nous a permis d'obtenir la liste de dossiers la plus exhaustive possible. 
Il est à noter que deux patientes correspondant aux critères d'inclusion ont été exclues car les feuilles d'anesthésie n'ont pas été retrouvées dans les dossiers médicaux.

Au centre hospitalier du Belvédère et au centre hospitalier des Feugrais, la liste des patientes a été facilement établie par le département d'information médicale, à partir du logiciel de codage diagnostique, avec les critères suivants : « révision utérine, délivrance artificielle ou extraction fœtale instrumentale " et " anesthésie générale en salle de naissance ». De rares dossiers ayant été codés par erreur selon ces critères n'ont pas été inclus.

\subsubsection{Elaboration du tableau de recueil de données}

Le tableau de recueil de données a été établi après lecture des dix premiers dossiers.

Les données recueillies comportaient :

- Une partie épidémiologique regroupant : l'âge des patientes, la poids, la taille et l'indice de masse corporelle (IMC), le terme de la grossesse, ainsi que la parité.

- Une partie regroupant les éléments relatifs au risque anesthésique : allergie, présence d'un reflux gastro-œsophagien, critères d'intubation difficile, facteurs de risque hémorragique (antécédent hémorragique ou trouble de la coagulation), score ASA.

- Une partie concernant les gestes obstétricaux: révision utérine, délivrance artificielle, extraction fœtale manuelle ou instrumentale, révision sous valve ou suture d'épisiotomie ou de déchirure périnéale, réalisés ou non dans un contexte d’hémorragie de la délivrance.

- Une partie concernant les techniques anesthésiques mises en œuvre : agents anesthésiques utilisés, technique de ventilation (intubation, ventilation spontanée au masque, ventilation manuelle au masque...), utilisation ou non d'un tampon gastrique, durée de l'anesthésie, association à une anesthésie loco-régionale ou non.

- Et enfin une partie concernant les complications liées à l'anesthésie : désaturation, réaction anaphylactique, intubation difficile, vomissement, inhalation.

\subsubsection{Sources des données}

L'ensemble des données a été recueilli à partir des dossiers médicaux.

Pour le CHU de Rouen et le centre hospitalier des Feugrais, il s'agissait d'un dossier médical uniquement manuscrit. 
Pour le centre hospitalier du Belvédère, il s'agissait d'un dossier médical manuscrit et informatique. En effet, dans ce centre de nombreuses données anesthésiques et obstétricales étaient disponibles sur le dossier informatique des patientes.

La plupart des données épidémiologiques et anesthésiques provenaient de la feuille d'anesthésie (manuscrite ou informatique). Les données obstétricales (geste réalisé, contexte obstétrical, existence éventuelle d'une hémorragie de la délivrance) provenaient principalement du partogramme tenu par les sages-femmes mais également, dans certains cas, de la feuille d'anesthésie.

Le dossier de suites de couches a également été étudié afin de s'assurer de l'absence de complication retardée (pneumopathie sur une micro-inhalation passée inaperçue au moment de l'anesthésie, par exemple).

\subsection{Analyse statistique}

Les données qualitatives étaient exprimées sous forme de pourcentages. Les données quantitatives étaient exprimées sous forme de moyennes (+/- écart-type) ou de médiane (intervalle interquartile). Le test de Student a été utilisé pour comparer deux paramètres quantitatifs, et le test du Chi 2 pour comparer deux paramètres qualitatifs.

Pour la comparaison des données entre les différents groupes, les données des deux centres hospitaliers périphériques ont été rassemblées et comparées aux données du CHU. Cela a permis d'obtenir deux groupes d'effectif comparable (62 patientes en périphérie versus 68 patientes au $\mathrm{CHU})$.

Pour rechercher une corrélation entre différents paramètres (facteurs influençant le choix d'une technique anesthésique par rapport à une autre) une analyse univariée puis multivariée a été réalisée. Pour l'analyse univariée, les tests du Chi 2 et du Fisher exact ont été utilisés. L'analyse multivariée a été réalisée selon un modèle de régression logistique. Pour l'analyse multivariée, seuls les critères ayant un seuil de significativité $p<0,2$ en analyse univariée ont été inclus.

Le niveau de significativité était fixé pour une valeur de $p<0,05$.

Les analyses statistiques ont été réalisées à partir des logiciels Prism V 5.0 (GraphPad Software, Inc, La Jolla, California, USA) et Stata V11 (StataCorp LP, College Station, Texas, USA), ainsi qu'à partir du site internet de la faculté de Paris 6 (Jussieu) : http://marne.u707.jussieu.fr/biostatgv/. 


\subsection{Aspects éthiques}

Les données utilisées ont été rendues anonymes et n'ont pas été stockées sur un logiciel partagé. Le département d'information médicale du CHU de Rouen a été informé de l'étude. Du fait du caractère purement rétrospectif et observationnel de l'étude (aucune intervention réalisée), l'avis du Comité de Protection des Personnes (CPP) n'était pas nécessaire. 


\section{Résultats}

\subsection{Inclusion}

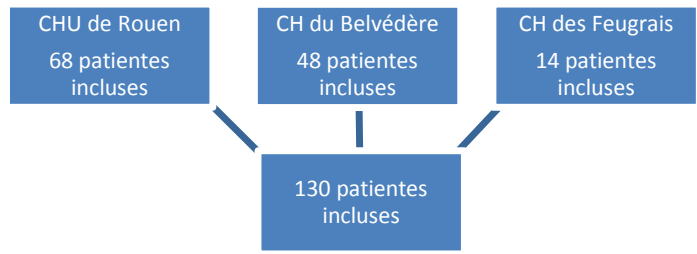

Après la sélection des dossiers selon la méthode décrite précédemment, le nombre de dossiers sélectionnés au CHU de Rouen étaient de 68. Au centre hospitalier du Belvédère, il était de 48 et au centre hospitalier des Feugrais de 14.

Au total, 130 patientes ont donc été incluses.

\subsection{Caractéristiques générales de la population}

\subsubsection{Données épidémiologiques}

Les caractéristiques épidémiologiques générales de la population sont décrites dans le tableau 1a. Deux groupes ont été comparés: le groupe des patientes provenant du CHU et le groupe des patientes provenant des centres hospitaliers périphériques ( $\mathrm{CH}$ du Belvédère et $\mathrm{CH}$ des Feugrais). II n'y avait pas de différence significative entre les groupes $\mathrm{CHU}$ et $\mathrm{CH}$ périphériques pour l'ensemble des critères étudiés hormis pour le terme de la grossesse, inférieur dans le groupe $\mathrm{CHU}$ de Rouen.

Tableau 1a : Caractéristiques générales de la population, épidémiologie

\begin{tabular}{|c|c|c|c|c|c|}
\hline & \multirow{2}{*}{$\begin{array}{c}\text { Total } \\
(\mathrm{N}=130)\end{array}$} & \multirow{2}{*}{$\begin{array}{l}\text { CHU Rouen } \\
(N=68)\end{array}$} & \multicolumn{2}{|c|}{ CH Périphériques ( $\mathrm{N}=62$ ) } & \multirow[b]{2}{*}{$\mathbf{p}$} \\
\hline & & & $\begin{array}{c}\text { CH Belvédère } \\
(N=48)\end{array}$ & $\begin{array}{c}\text { CH Feugrais } \\
(N=14)\end{array}$ & \\
\hline Age, moyenne +/- DS, année & $29,9 \pm 5,3$ & $29,7 \pm 5,7$ & $31 \pm 4,5$ & $27,4 \pm 5$ & 0,60 \\
\hline Taille, moyenne +/- DS, cm & $165 \pm 6,3$ & $165 \pm 6,3$ & $164 \pm 5,9$ & $167 \pm 7,9$ & 0,86 \\
\hline Poids, moyenne +/- DS, kg & $76 \pm 13,2$ & $76 \pm 14$ & $75,4 \pm 12,5$ & $78,7 \pm 11,7$ & 0,96 \\
\hline BMI, moyenne +/- DS, kg/m² & $28,1 \pm 4,5$ & $28,1 \pm 4,8$ & $27,8 \pm 4,2$ & $28,5 \pm 4,2$ & 0,85 \\
\hline Terme, moyenne +/- DS, SA & $38,4+/-4,1$ & $37,3+/-4,8$ & $39,8+/-2,9$ & $39,2+/-2$ & 0,0007 \\
\hline Parité, moyenne +/- DS & $2,4+/-1,2$ & $2,5+/-1,4$ & $2,3+/-0,9$ & $2,3+/-1,2$ & 0,31 \\
\hline
\end{tabular}




\subsubsection{Données anesthésiques}

Les informations issues de la consultation d'anesthésie sont résumées dans les tableaux 1b et 1c.

II n'y avait pas de différence significative entre le groupe $\mathrm{CHU}$ et le groupe $\mathrm{CH}$ périphériques.

Tableau 1b : Caractéristiques générales de la population, données anesthésiques

\begin{tabular}{|c|c|c|c|c|c|}
\hline & \multirow[b]{2}{*}{$\begin{array}{c}\text { Total } \\
(\mathrm{N}=130)\end{array}$} & \multirow[b]{2}{*}{$\begin{array}{l}\text { CHU Rouen } \\
\qquad(\mathrm{N}=68)\end{array}$} & \multicolumn{2}{|c|}{ CH Périphériques ( $N=62$ ) } & \multirow[b]{2}{*}{$\mathbf{p}$} \\
\hline & & & $\begin{array}{l}\text { CH Belvédère } \\
(\mathrm{N}=48)\end{array}$ & $\begin{array}{l}\text { CH Feugrais } \\
\qquad(\mathrm{N}=14)\end{array}$ & \\
\hline Score ASA, médiane (25\%-75\%) & $1(1-2)$ & $1(1-2)$ & $1(1-1)$ & $1(1-1)$ & $\mathrm{nc}$ \\
\hline RGO, n (\%) & $25(19)$ & $17(25)$ & $3(6)$ & $5(38)$ & 0,08 \\
\hline Allergie, n (\%) & $18(14)$ & $11(16)$ & $7(15)$ & 0 & 0,42 \\
\hline Risque hémorragique, n (\%) & $20(15)$ & $13(19)$ & $7(15)$ & 0 & 0,22 \\
\hline Critère d'intubation difficile, $n$ (\%) & $18(14)$ & $13(19)$ & $5(10)$ & 0 & 0,07 \\
\hline
\end{tabular}

nc : non calculable

RGO : reflux gastro-œsophagien

L'ensemble des patientes avaient un score ASA inférieur ou égal à 2.

Le critère "risque hémorragique " regroupait: les antécédents hémorragiques marquant (notamment antécédent d'hémorragie du post-partum), les troubles de l'hémostase primaire et secondaire.

Tableau 1c : Critères d'intubation difficile

\begin{tabular}{|c|c|c|c|c|c|}
\hline & \multirow[b]{2}{*}{$\begin{array}{c}\text { Total } \\
(\mathrm{N}=130)\end{array}$} & \multirow[b]{2}{*}{$\begin{array}{l}\text { CHU Rouen } \\
\qquad(N=68)\end{array}$} & \multicolumn{2}{|c|}{ CH périphériques ( $N=62$ ) } & \multirow[b]{2}{*}{ p } \\
\hline & & & $\begin{array}{c}\text { CH Belvédère } \\
(N=48)\end{array}$ & $\begin{array}{l}\text { CH Feugrais } \\
(\mathrm{N}=14)\end{array}$ & \\
\hline Au moins 1 critère, n (\%) & $18(14)$ & $13(19)$ & $5(10)$ & 0 & 0,07 \\
\hline Mallampati > 2, n (\%) & $5(4)$ & $5(8)$ & 0 & 0 & nc \\
\hline Ouverture de bouche $<35 \mathrm{~mm}, \mathrm{n}(\%)$ & $2(1,5)$ & $2(3)$ & 0 & 0 & nc \\
\hline ATCD d'IOT difficile, $n$ (\%) & 0 & 0 & 0 & 0 & nc \\
\hline IMC > 35 kg/m², n (\%) & $11(8)$ & $6(8)$ & $5(10)$ & 0 & 0,9 \\
\hline Autre, n (\%) & $2(2)$ & $2(3)$ & 0 & 0 & $\mathrm{nc}$ \\
\hline
\end{tabular}


Le critère " distance thyro-mentonnière » n'a pas été pris en compte : jusqu'en 2010 il ne figurait pas sur les feuilles d'anesthésie et n'était donc qu'exceptionnellement mentionné.

Deux patientes étaient considérées comme à risque d'intubation difficile en raison d'autres critères : une macroglossie pour l'une, et pour l'autre un antécédent de chirurgie de la mâchoire pour luxation récidivante.

\subsection{Contexte obstétrical}

Le contexte obstétrical dans lequel l'anesthésie générale a été réalisée est présenté dans les tableaux $2 a$ et $2 b$.

Le tableau 2a permet de résumer l'ensemble des gestes pour lesquels une anesthésie générale a été nécessaire.

Le tableau $2 \mathrm{~b}$ montre la prévalence de l'hémorragie du post-partum dans la cohorte.

II n'y avait pas de différence significative entre le groupe $\mathrm{CHU}$ de Rouen et le groupe $\mathrm{CH}$ périphériques, hormis pour la catégorie "suture périnéale ", qui était plus pratiquée dans le groupe $\mathrm{CH}$ périphériques.

Tableau 2a : Gestes réalisés

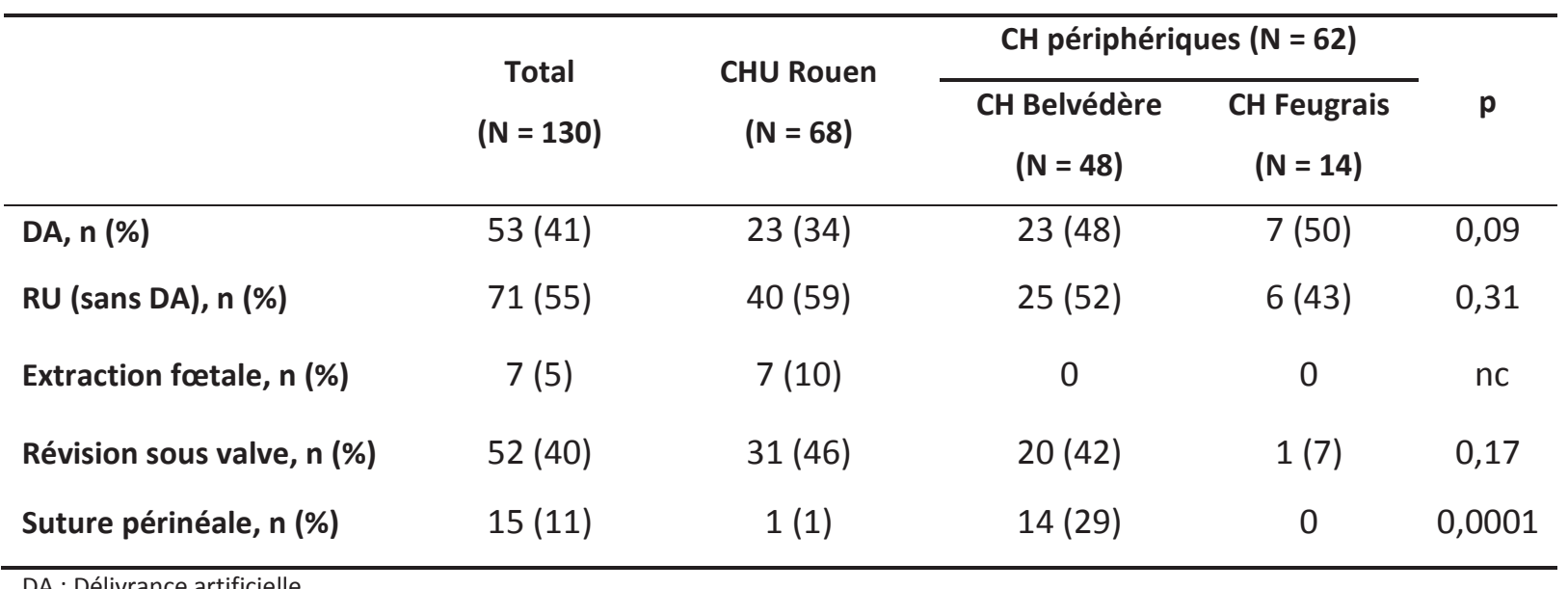

RU : Révision utérine

nc : non calculable

La catégorie "suture périnéale » regroupe les sutures d'épisiotomie ou de déchirures périnéales n’ayant pu être réalisées sous anesthésie locale.

La catégorie " extraction fœtale " regroupe les manœuvres d'extraction instrumentale, par forceps, ventouse ou spatule $(n=4)$ et les manœuvres de version-grande extraction sur le deuxième jumeau $(n=3)$. En effet, ces deux techniques sont comparables sur le plan anesthésique puisque, dans les 
deux cas, l'utérus est toujours gravide au moment de l'anesthésie générale, et que, dans les deux cas, il s'agit d'un geste d'extraction fœtale, considéré comme plus invasif que les autres gestes réalisés. Aucun cas d'extraction fœtale sous anesthésie générale n'a été recensé dans les centres hospitaliers périphériques.

Plusieurs gestes étaient souvent associés chez une même patiente, notamment en raison du grand nombre d'hémorragies du post-partum, pour lesquelles l'algorithme de prise en charge implique la réalisation de plusieurs gestes différents.

Tableau 2b : Prévalence de l'hémorragie du post-partum dans la cohorte

\begin{tabular}{|c|c|c|c|c|c|}
\hline & \multirow[b]{2}{*}{$\begin{array}{c}\text { Total } \\
(N=130)\end{array}$} & \multirow[b]{2}{*}{$\begin{array}{l}\text { CHU Rouen } \\
\qquad(\mathrm{N}=68)\end{array}$} & \multicolumn{2}{|c|}{$\mathrm{CH}$ périphériques $(\mathrm{N}=62)$} & \multirow[b]{2}{*}{$\mathbf{p}$} \\
\hline & & & $\begin{array}{c}\text { CH Belvédère } \\
(\mathrm{N}=48)\end{array}$ & $\begin{array}{l}\text { CH Feugrais } \\
(\mathrm{N}=14)\end{array}$ & \\
\hline Hémorragie du post-partum, n (\%) & $73(56)$ & 40 (59) & $30(62)$ & $3(21)$ & 0,12 \\
\hline
\end{tabular}

\subsection{Prise en charge anesthésique}

\subsubsection{Prise en charge des voies aériennes supérieures}

Le tableau 3 montre les taux d'intubation et de ventilation au masque, sur l'ensemble de la cohorte et dans chacun des trois centres.

Au total, 27 patientes (soit $21 \%$ de la cohorte) ont été intubées, 101 patientes (soit 78\%) ont été laissées en ventilation spontanée au masque facial, et 2 patientes (soit 1\%) ont été ventilées manuellement au masque facial.

II existait une disparité des pratiques, statistiquement significative, entre le $\mathrm{CHU}$ de Rouen et les $\mathrm{CH}$ périphériques.

Tableau 3 : Prise en charge des voies aériennes supérieures

\begin{tabular}{|c|c|c|c|c|c|}
\hline & \multirow{2}{*}{$\begin{array}{c}\text { Total } \\
(\mathrm{N}=130)\end{array}$} & \multirow{2}{*}{$\begin{array}{l}\text { CHU Rouen } \\
(\mathrm{N}=68)\end{array}$} & \multicolumn{2}{|c|}{$\mathrm{CH}$ périphériques ( $\mathrm{N}=62$ ) } & \multirow[b]{2}{*}{$\mathbf{p}$} \\
\hline & & & $\begin{array}{c}\text { CH Belvédère } \\
(\mathrm{N}=48)\end{array}$ & $\begin{array}{l}\text { CH Feugrais } \\
\qquad(\mathrm{N}=14)\end{array}$ & \\
\hline IOT, n (\%) & $27(21)$ & $21(31)$ & $5(10)$ & $1(7)$ & 0,003 \\
\hline VS au masque, $n(\%)$ & $101(78)$ & $46(68)$ & $42(88)$ & $13(93)$ & 0,004 \\
\hline $\begin{array}{l}\text { Ventilation manuelle } \\
\text { au masque, } \mathrm{n}(\%)\end{array}$ & $2(1)$ & $1(1)$ & $1(2)$ & 0 & $\mathrm{nc}$ \\
\hline
\end{tabular}


Les figures $1 \mathrm{a}, 1 \mathrm{~b}$ et $1 \mathrm{c}$ permettent de visualiser la répartition des différentes techniques de prise en charge des voies aériennes supérieures sur l'ensemble de la cohorte et dans les différents centres.

Figure 1a : Prise en charge des voies aériennes supérieures dans l'ensemble de la cohorte

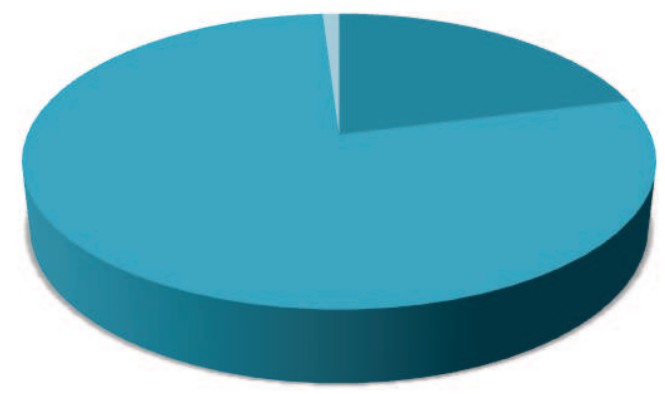

- IOT

Ventilation spontanée au masque

Ventilation manuelle au masque

Figure 1b : Prise en charge des voies aériennes supérieures au CHU

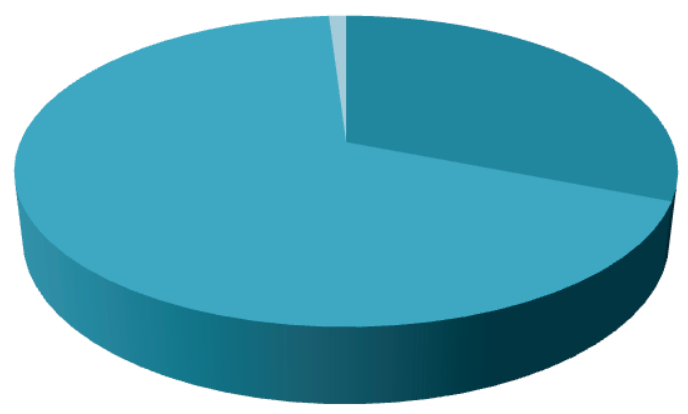

IOT

- Ventilation spontanée au masque

Ventilation manuelle au masque

Figure 1c : Prise en charge des voies aériennes supérieures dans les $\mathrm{CH}$ périphériques
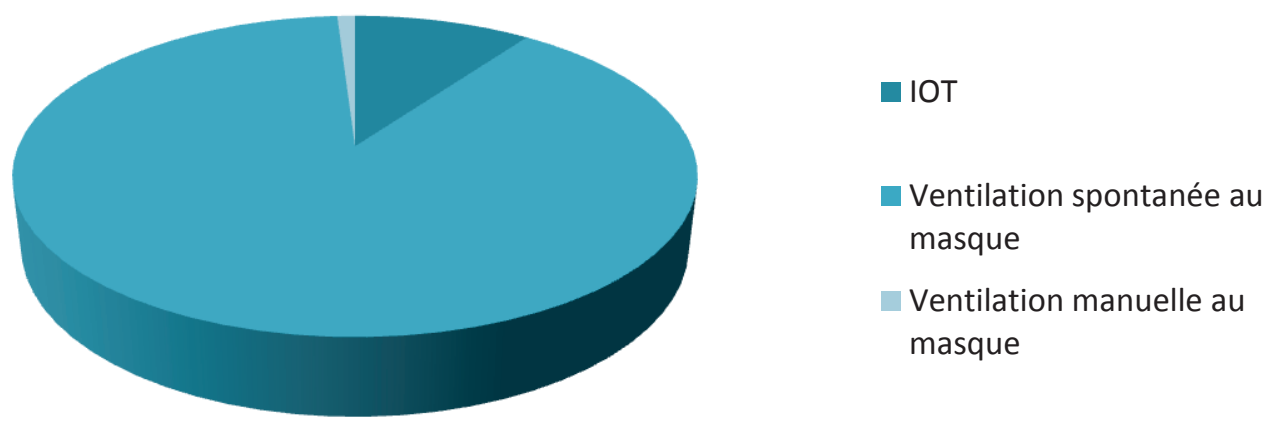


\subsubsection{Agents anesthésiques}

Lorsque les patientes étaient intubées, une induction en séquence rapide était réalisée dans $100 \%$ des cas, par une association thiopental/succinylcholine, propofol/succinylcholine ou kétamine/succinylcholine. L'entretien était réalisé par différents agents (hypnotiques et/ou antalgiques), utilisés seuls ou, le plus souvent, en association.

Lorsque les patientes n'étaient pas intubées, ces mêmes agents étaient utilisés, à moindre dose.

Les tableaux $4 \mathrm{a}$ et $4 \mathrm{~b}$ montrent les différents protocoles utilisés pour l'anesthésie générale, selon les modalités de ventilation des patientes.

Tableau 4a : Agents anesthésiques utilisés en cas d'IOT

\begin{tabular}{|c|c|c|c|c|c|c|c|}
\hline & \multicolumn{3}{|c|}{ Induction } & \multicolumn{4}{|c|}{ Entretien } \\
\hline & $\begin{array}{c}\text { Thiopental + } \\
\text { succinylcholine }\end{array}$ & $\begin{array}{c}\text { Propofol + } \\
\text { succinylcholine }\end{array}$ & $\begin{array}{c}\text { Kétamine + } \\
\text { succinylcholine }\end{array}$ & Propofol & Kétamine & Alfentanil & Autre* \\
\hline $\begin{array}{c}\text { IOT }(n=27), \\
n(\%)\end{array}$ & $13(48)$ & $13(48)$ & $1(4)$ & $11(41)$ & $4(15)$ & $12(44)$ & $4(15)$ \\
\hline
\end{tabular}

* sufentanil $(\mathrm{n}=2)$, remifentanil $(\mathrm{n}=1)$, midazolam $(\mathrm{n}=1)$

IOT : intubation oro-trachéale

Tableau 4b : Agents utilisés en cas de ventilation au masque

\begin{tabular}{|c|c|c|c|c|c|c|}
\hline & Propofol & Kétamine & $\begin{array}{l}\text { Propofol + } \\
\text { Kétamine }\end{array}$ & $\begin{array}{l}\text { Propofol + } \\
\text { Alfentanil }\end{array}$ & $\begin{array}{l}\text { Kétamine + } \\
\text { Midazolam }\end{array}$ & Autres* \\
\hline $\begin{array}{l}\text { Ventilation spontanée au } \\
\text { masque ( } \mathrm{n}=101), \mathrm{n}(\%)\end{array}$ & 19 (19) & 35 (35) & 19 (19) & $10(10)$ & $5(5)$ & $13(13)$ \\
\hline $\begin{array}{l}\text { Ventilation manuelle au } \\
\text { masque }(n=2), n(\%)\end{array}$ & & & $1(50)$ & & & $1(50)$ \\
\hline
\end{tabular}

* Kétamine + Alfentanil $(n=4)$, Kétamine + Alfenatnil + Hypnovel $(n=2)$, Ketamine + Sufentanil + Midazolam $(n=1)$, Kétamine + Propofol + Midazolam $(n=4)$, Kétamine + Propofol + Alfentanil + Midazolam $(n=1)$, Propofol + Sufenta $(n=2)$

La succinylcholine était utilisée à la dose de $100 \mathrm{mg}( \pm 24)$ soit $1,3 \mathrm{mg} / \mathrm{kg}$ et le thiopental à la dose de $435 \mathrm{mg}( \pm 98)$ soit $5,7 \mathrm{mg} / \mathrm{kg}$. Le propofol était utilisé à la dose de $220 \mathrm{mg}( \pm 107)$ soit $2,9 \mathrm{mg} / \mathrm{kg}$ chez les patientes intubées, versus $180 \mathrm{mg}( \pm 82)$ soit $2,4 \mathrm{mg} / \mathrm{kg}$ chez les patientes non intubées $(p=0,15)$. La kétamine était utilisée à la dose de $55 \mathrm{mg}( \pm 34)$ soit $0,7 \mathrm{mg} / \mathrm{kg}$ chez les patientes intubées, versus $54 \mathrm{mg}( \pm 25)$ soit $0,7 \mathrm{mg} / \mathrm{kg}$ chez les patientes non intubées $(p=0,9)$. L'alfentanil était utilisé à la dose 
de $1,8 \mathrm{mg}( \pm 0,5)$ soit $23 \mu \mathrm{g} / \mathrm{kg}$ chez les patientes intubées, versus $1,1 \mathrm{mg}( \pm 0,3)$ soit $14 \mu \mathrm{g} / \mathrm{kg}$ chez les patientes non intubées $(p=0,02)$. Le midazolam était utilisé à la dose de $5 \mathrm{mg}$ soit $0,07 \mathrm{mg} / \mathrm{kg}$ chez les patientes intubées versus $2 \mathrm{mg}( \pm 1)$ soit $0,03 \mathrm{mg} / \mathrm{kg}$ chez les patientes non intubées ( $p$ non calculable). Le sufentanil était utilisé à la dose de $12,5 \mu \mathrm{g}( \pm 3,5)$ soit $0,16 \mu \mathrm{g} / \mathrm{kg}$ chez les patientes intubées versus $12 \mu \mathrm{g}( \pm 3)$ soit $0,15 \mu \mathrm{g} / \mathrm{kg}$ chez les patientes non intubées $(p=0,8)$.

\subsubsection{Tamponnement de l’acidité gastrique}

Nous n'avons obtenu que très peu de données concernant la délivrance ou non d'un tampon de l'acidité gastrique (données manquantes) puisqu'au total, selon les informations retrouvées dans les dossiers, seules $18 \%$ des patientes en auraient bénéficié. Ce taux était de $18 \%$ au CHU de Rouen, de $4 \%$ au $\mathrm{CH}$ du Belvédère et de $64 \%$ au $\mathrm{CH}$ des Feugrais.

\subsubsection{Durée de l'anesthésie}

La durée de l'anesthésie générale était en moyenne de 28 minutes ( \pm 17$)$. Au CHU de Rouen elle était de 31 minutes ( \pm 19 ), au $\mathrm{CH}$ du Belvédère elle était de 27 minutes $( \pm 15$ ) et au $\mathrm{CH}$ des Feugrais elle était de 19 minutes ( \pm 9). La durée de l’anesthésie générale n'était pas plus importante en cas d'hémorragie du post-partum (29 minutes vs 27 minutes en l'absence d'hémorragie du post-partum).

\subsubsection{Anesthésie loco-régionale}

Parmi les patientes incluses dans l'étude, 4 (soit 3\%) avaient bénéficié d'une anesthésie locorégionale (anesthésie péridurale ou rachianesthésie). Dans 2 des 4 cas, l'analgésie s'était avérée insuffisante pour le geste obstétrical, malgré la réalisation préalable d’une anesthésie péridurale (pour le travail). Dans le $3^{\text {ème }}$ cas, l'anesthésie générale avait été faite après échec d'une rachianesthésie. Enfin, dans le dernier cas, la patiente bénéficiait déjà d'une anesthésie péridurale efficace mais, devant la gravité de l'hémorragie du post-partum qu'elle présentait, elle avait finalement été intubée. 


\subsection{Facteurs influençant le choix de la technique anesthésique}

\subsubsection{Analyse univariée}

\subsubsection{Taux d'intubation selon le centre}

Comme il a été montré précédemment, le taux d'intubation était plus important au CHU de Rouen par rapport aux $\mathrm{CH}$ périphériques, puisqu'il était de $21 \%$ versus $10 \%(p=0,003)$.

\subsubsection{Taux d'intubation selon le contexte obstétrical}

Le taux d'intubation oro-trachéale variait selon le geste obstétrical réalisé, et pour un même geste, selon le centre. Le tableau 5 a et la figure 2a présentent les différents taux d'intubation en fonction du geste réalisé, dans chaque centre.

Toutes les patientes ayant eu une extraction fœtale avaient été intubées. A l'inverse, aucune des patientes endormies pour la réalisation d'une suture périnéale n'avait été intubée.

Tableau 5a : Taux d'intubation en fonction du geste réalisé

\begin{tabular}{lcccc}
\hline & Total $(\mathbf{N}=130)$ & CHU (N = 68) & \multicolumn{2}{c}{ CH périphériques (N = 62) } \\
\cline { 4 - 5 } & & & CH Belvédère (N = 48) & CH Feugrais (N = 14) \\
\hline DA, $\mathbf{n} / \mathbf{N}(\%)$ & $9 / 53(17)$ & $5 / 23(22)$ & $3 / 23(13)$ & $1 / 7(14)$ \\
RU (sans DA), $\mathbf{n / N}(\%)$ & $14 / 71(20)$ & $12 / 40(30)$ & $2 / 25(8)$ & $0 / 6(0)$ \\
Extraction fœtale, $\mathbf{n} / \mathbf{N}(\%)$ & $7 / 7(100)$ & $7 / 7(100)$ & 0 & 0 \\
Révision sous valve, $\mathbf{n / N}(\%)$ & $12 / 52(23)$ & $9 / 31(29)$ & $3 / 20(15)$ & $0 / 1(0)$ \\
Suture périnéale, $\mathbf{n} / \mathbf{N}(\%)$ & $0 / 15(0)$ & $0 / 1(0)$ & $0 / 14(0)$ & 0 \\
\hline
\end{tabular}

$\mathrm{n}$ : nombre de patientes intubées parmi celles ayant bénéficié du geste étudié

$\mathrm{N}$ : nombre de patientes ayant bénéficié du geste étudié

DA : Délivrance artificielle

RU : Révision utérine 
Figure 2a : Taux d'intubation en fonction du geste réalisé et selon le centre

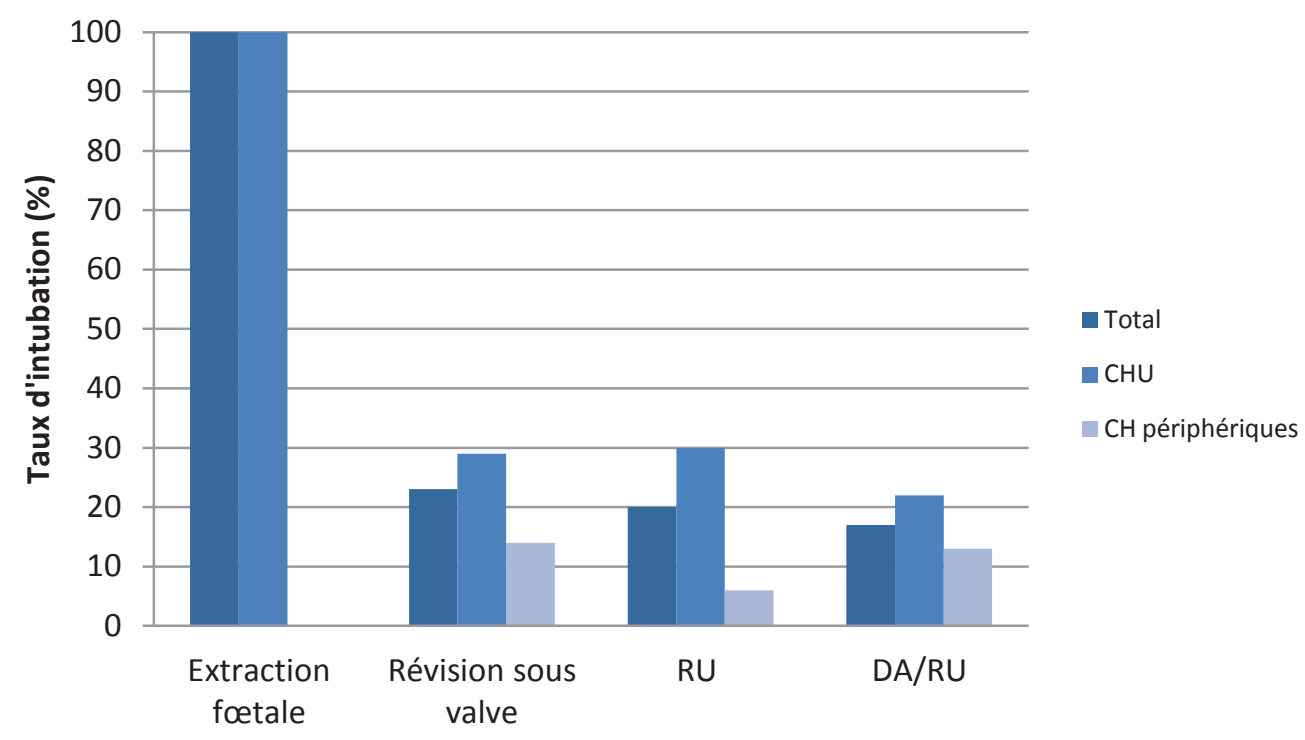

DA : Délivrance artificielle

RU : Révision utérine

Le taux d'intubation pour extraction fœtale dans les $\mathrm{CH}$ périphériques n'est pas représenté puisqu'aucune extraction fœtale sous anesthésie générale n’a été pratiquée dans les $\mathrm{CH}$ périphériques.

L'extraction fœtale était associée de façon très significative à un plus fort taux d'intubation $(p<$ $0,0001)$ puisque celle-ci était pratiquée dans $100 \%$ des cas. 
Le taux d'intubation variait également selon la présence ou non d'une hémorragie du post-partum. Cela est représenté dans le tableau $5 \mathrm{~b}$ et la figure $2 \mathrm{~b}$.

Tableau 5b : Taux d'intubation en cas d'hémorragie du post-partum

\begin{tabular}{ccccc}
\hline & Total $(\mathbf{N}=130)$ & CHU $(\mathbf{N}=68)$ & \multicolumn{2}{c}{ CH périphériques $(\mathbf{N}=62)$} \\
\cline { 3 - 4 } & & & CH Belvédère $(\mathbf{N}=48)$ & CH Feugrais (N = 14) \\
\hline HPP, $n / N(\%)$ & $20 / 73(27)$ & $15 / 40(37)$ & $4 / 30(13)$ & $1 / 3(33)$ \\
\hline
\end{tabular}

$\mathrm{n}$ : nombre de patientes intubées parmi celles présentant une hémorragie du post-partum

$\mathrm{N}$ : nombre de patientes présentant une hémorragie du post-partum

HPP : Hémorragie du post-partum

Figure $2 b$ : Taux d'intubation en fonction de la présence ou non d'une hémorragie du post-partum

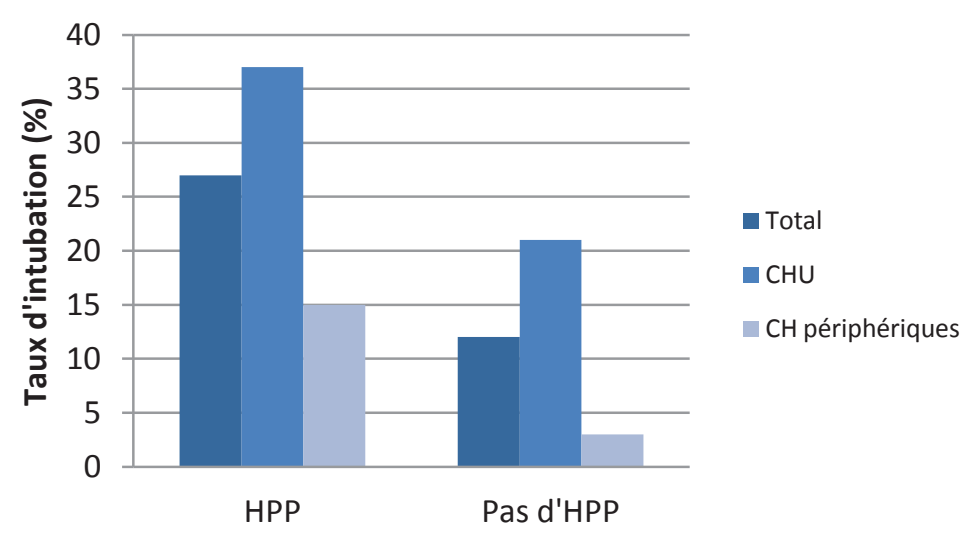

HPP : Hémorragie du post-partum

La présence d'une hémorragie du post-partum était associée de façon significative à un plus fort taux d'intubation : $27 \%$ en cas d'hémorragie du post-partum versus $12 \%$ en l'absence d'hémorragie du post-partum $(p=0,03)$.

\subsubsection{Taux d'intubation selon le terrain des patientes}

Le taux d'intubation variait selon le contexte clinique obstétrical comme il vient d'être montré, mais aussi en fonction du terrain des patientes. Le tableau 6 et la figure 3 mettent en évidence ces différences de pratiques selon la présence ou non d'un RGO, d'un trouble de l'hémostase, d'un IMC $\geq$ $35 \mathrm{~kg} / \mathrm{m}^{2}$, de facteurs de risque d'intubation difficile ou d'antécédent allergique. 
Tableau 6 : Taux d'intubation en fonction du terrain des patientes

\begin{tabular}{|c|c|c|c|c|}
\hline & \multirow{2}{*}{ Total $(N=130)$} & \multirow{2}{*}{$\mathrm{CHU}(\mathrm{N}=68)$} & \multicolumn{2}{|c|}{$\mathrm{CH}$ périphériques $(\mathrm{N}=62)$} \\
\hline & & & CH Belvédère ( $\mathrm{N}=48)$ & CH Feugrais $(\mathrm{N}=14)$ \\
\hline \multicolumn{5}{|l|}{$R G O$} \\
\hline Oui, n/N (\%) & $9 / 25(36)$ & $9 / 17(53)$ & $0 / 3(0)$ & $0 / 5(0)$ \\
\hline Non, n/N (\%) & 18/105 (17) & $12 / 51(23)$ & $5 / 45(11)$ & $1 / 9(11)$ \\
\hline \multicolumn{5}{|l|}{ Trouble de l'hémostase } \\
\hline Oui, n/N (\%) & $5 / 20(25)$ & $3 / 13(23)$ & 2/7 (29) & 0 \\
\hline Non, n/N (\%) & $22 / 110(20)$ & 18/55 (33) & $3 / 41(7)$ & $1 / 14(7)$ \\
\hline \multicolumn{5}{|l|}{ IMC > $35 \mathrm{~kg} / \mathrm{m}^{2}$} \\
\hline Oui, n/N (\%) & $5 / 11(45)$ & $4 / 6(67)$ & $1 / 5(20)$ & 0 \\
\hline Non, n/N (\%) & $22 / 119(18)$ & $17 / 62(27)$ & 4/43 (9) & $1 / 14(7)$ \\
\hline \multicolumn{5}{|l|}{ Facteur de risque d'IOT } \\
\hline \multicolumn{5}{|l|}{ difficile } \\
\hline Oui, n/N (\%) & $6 / 18(33)$ & $5 / 13(38)$ & $1 / 5(20)$ & 0 \\
\hline Oui sauf IMC $\geq 35, \mathrm{n} / \mathrm{N}$ & $1 / 7(14)$ & $1 / 8(12)$ & 0 & 0 \\
\hline \multicolumn{5}{|l|}{ (\%) } \\
\hline Non, n/N (\%) & $21 / 112(19)$ & $16 / 55(29)$ & $4 / 43(9)$ & $1 / 14(7)$ \\
\hline \multicolumn{5}{|l|}{ Antécédent allergique } \\
\hline Oui, n/N (\%) & $4 / 18(22)$ & $4 / 11(36)$ & $0 / 7(0)$ & 0 \\
\hline Non, n/N (\%) & $23 / 112(20)$ & $17 / 57(30)$ & $5 / 41(12)$ & $1 / 14(7)$ \\
\hline \multicolumn{5}{|c|}{$\mathrm{n}:$ nombre de patientes intubées parmi celles présentant (ou non) le critère } \\
\hline \multicolumn{5}{|c|}{$N$ : nombre de patientes présentant (ou non) le critère } \\
\hline \multicolumn{5}{|l|}{ RGO : reflux gastro-œsophagien } \\
\hline IMC : indice de masse corpore & & & & \\
\hline
\end{tabular}


Figure 3 : Taux d'intubation en fonction du terrain des patientes

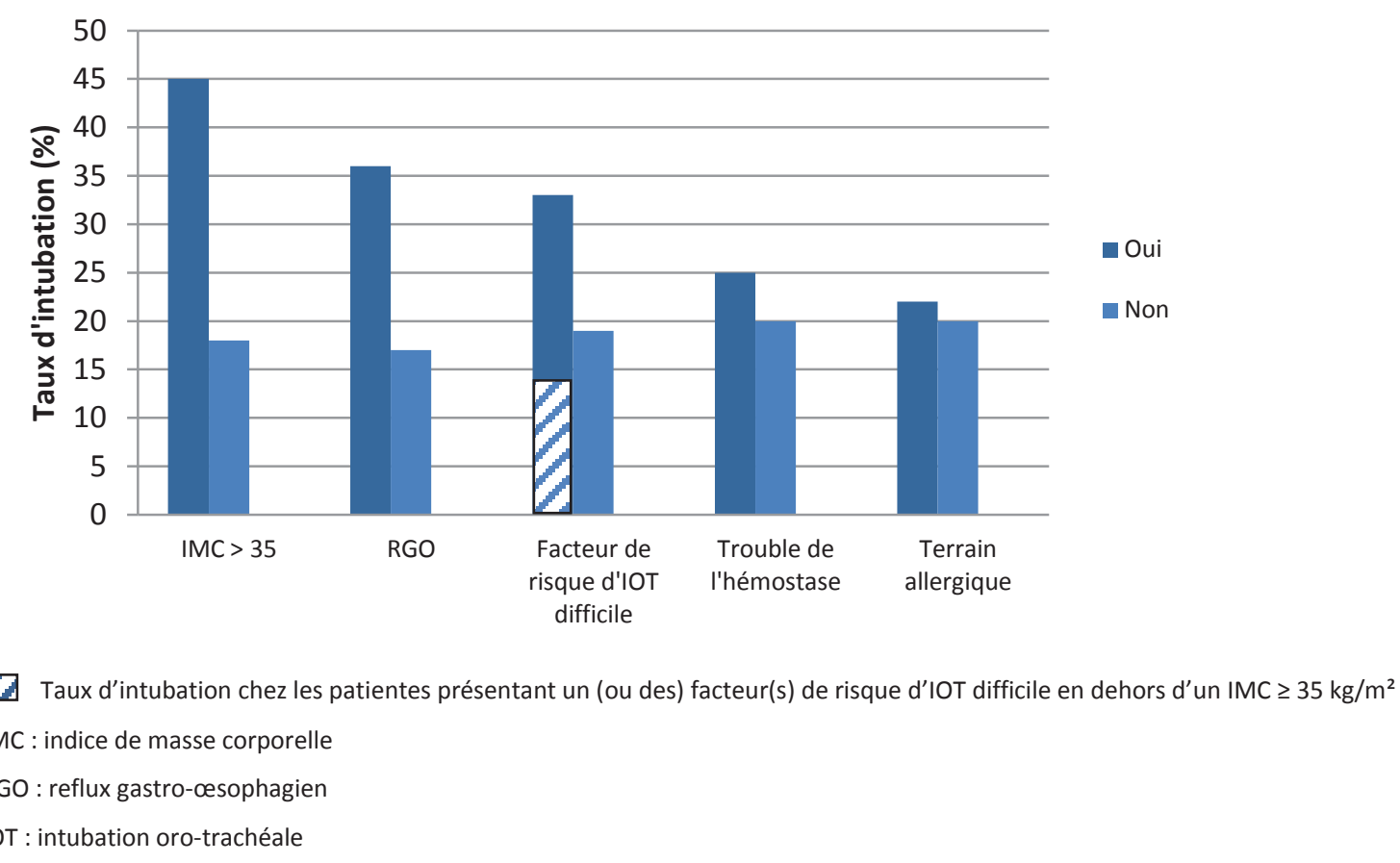

Parmi les différents facteurs étudiés, la présence d'un $I M C \geq 35 \mathrm{~kg} / \mathrm{m}^{2}$ et la présence d'un RGO étaient associées à un plus fort taux d'intubation. En effet, en cas de RGO, 36\% des patientes étaient intubées versus $17 \%$ en l'absence de RGO $(p=0,04)$ et en cas $d^{\prime} I M C \geq 35 \mathrm{~kg} / \mathrm{m}^{2}, 45 \%$ des patientes étaient intubées versus $18 \%$ en cas $d^{\prime} I M C<35 \mathrm{~kg} / \mathrm{m}^{2}(p=0,03)$. En revanche, la présence de troubles de I'hémostase, d'antécédents allergiques, ou de facteurs de risque d'intubation difficile, n'influençaient pas la décision (respectivement : $p=0,6 ; p=0,9 ; p=0,16$ ).

\subsubsection{Analyse multivariée}

D’après l'analyse univariée, les facteurs suivants ressortaient comme étant associés à un plus fort taux d'intubation : le site du CHU de Rouen, la réalisation d'une extraction fœtale, la présence d'une hémorragie de la délivrance, la présence d'un RGO, la présence d'un $I M C \geq 35 \mathrm{~kg} / \mathrm{m}^{2}$.

Afin d'approfondir l'analyse de ces facteurs, une analyse multivariée selon un modèle de régression logistique a été réalisée. Ainsi, en plus de l'extraction fœetale (critère n'ayant pu être intégré dans l'analyse multivariée car associé à un taux d'intubation de 100\%), l'analyse multivariée a permis de montrer que seuls I'hémorragie de la délivrance et $I^{\prime} I M C \geq 35 \mathrm{~kg} / \mathrm{m}^{2}$ étaient significativement associés à un taux d'intubation plus élevé, avec un odd ratio respectivement à 14,3 pour l'hémorragie de la délivrance (Intervalle de confiance à $95 \%[1,8-114,8],(p=0,01)$ ) et 6,1 pour l'IMC $\geq 35 \mathrm{~kg} / \mathrm{m}^{2}$ 
(Intervalle de confiance à 95\% [1,3-28], $(p=0,02))$. Les autres critères n'apparaissaient plus comme étant significativement corrélés à un plus fort taux d'intubation.

\subsection{Complications}

Deux complications sont survenues sur l'ensemble de la cohorte (soit dans 1,5\% des cas), toutes deux au $\mathrm{CHU}$ de Rouen, et dans les deux cas chez des patientes intubées. Le tableau 7 résume ces complications.

Tableau 7 : Complications chez les patientes intubées et non intubées

\begin{tabular}{lcccc}
\hline & Inhalation & Vomissement & $\begin{array}{c}\text { IOT } \\
\text { difficile }\end{array}$ & Anaphylaxie \\
\hline IOT $(n=27), n(\%)$ & $1(4)$ & $1(4)$ & 0 & 0 \\
$\begin{array}{l}\text { Ventilation au masque (spontanée ou manuelle) }(n= \\
\text { 103), } n(\%)\end{array}$ & 0 & 0 & 0 & 0 \\
\hline
\end{tabular}

IOT : intubation oro-trachéale

Dans le premier cas, il s'agissait d'un épisode de vomissement au moment de l'intubation. Cet épisode ne s'est pas compliqué de pneumopathie d'inhalation (évolution parfaitement favorable en suite de couches, sans complication respiratoire mentionnée). Pour le deuxième cas il s'agissait peutêtre d'une micro-inhalation survenue au moment de l'induction puisque la patiente a présenté des sécrétions sales en fin de geste (sans qu'aucun épisode de vomissement n'ait été visualisé), ce qui a conduit l'anesthésiste à introduire un traitement antibiotique par amoxicilline/acide clavulanique. La patiente n’a pas présenté de défaillance respiratoire par la suite.

Aucune complication n'est survenue chez les patientes non intubées. 


\section{Discussion}

\subsection{Synthèse des principaux résultats}

Cette étude rétrospective a permis d'analyser précisément les pratiques anesthésiques en cas d'anesthésie générale en fin de travail, dans trois centres hospitaliers de la région Haute-Normandie.

Cent trente patientes ayant nécessité une anesthésie générale en salle de naissance ont été incluses. Parmi les 130 patientes incluses, 21\% étaient intubées : 31\% au CHU de Rouen et $10 \%$ dans les $\mathrm{CH}$ périphériques du Belvédère et des Feugrais ( $p>0,05$ en analyse multivariée). Toutes les patientes intubées avaient bénéficié d'une induction en séquence rapide. Les patientes non intubées étaient laissées en ventilation spontanée au masque facial pour la plupart (78\%) et une aide par ventilation manuelle au masque facial avait été rapportée chez 2 patientes (1\%).

Les gestes réalisés étaient : une délivrance artificielle ( $41 \%$ des cas), une révision utérine $(55 \%$ des cas), une extraction fœtale ( $5 \%$ des cas), une révision sous valve ( $40 \%$ des cas) ou une suture périnéale ( $11 \%$ des cas). Cinquante-six pour-cent des patientes présentaient une hémorragie du postpartum.

La corrélation entre le taux d'intubation et le contexte clinique obstétrical d'une part et le terrain des patientes d'autre part a été étudiée par le biais d'une analyse uni- puis multivariée. En analyse univariée les critères suivants semblaient être associés à un plus fort taux d'intubation : prise en charge au CHU de Rouen (versus les $\mathrm{CH}$ périphériques) ( $p=0,003)$, contexte d'hémorragie du postpartum au moment de la prise en charge anesthésique $(p=0,03)$, réalisation d'une extraction fotale $(p<0,0001)$, présence d'un RGO $(p=0,04)$, présence d'un IMC $\geq 35 \mathrm{~kg} / \mathrm{m}^{2}(p=0,03)$.

Cependant, en plus de l'extraction fœtale (pour laquelle l'intubation est systématique), en analyse multivariée, seuls I'hémorragie du post-partum et $\mathrm{I}^{\prime} I M C \geq 35 \mathrm{~kg} / \mathrm{m}^{2}$ étaient associés à un plus fort taux d'intubation avec un odd ratio à 14,3 pour l'hémorragie de la délivrance (Intervalle de confiance à $95 \%[1,8-114,8],(p=0,01))$ et 6,1 pour l'IMC $\geq 35 \mathrm{~kg} / \mathrm{m}^{2}$ (Intervalle de confiance à $95 \%$ [1,3-28], ( $p$ $=0,02)$.

Deux complications sont survenues (7\%), toutes deux chez des patientes intubées : un épisode de vomissement à l'induction non compliqué de pneumopathie d'inhalation, et une probable pneumopathie d'inhalation non grave, sans épisode de vomissement préalable. Aucune complication n'est survenue chez les patientes non intubées. 


\subsection{Commentaires}

\subsubsection{Comparaison avec les données de la littérature}

Le taux d'intubation de $21 \%$ retrouvé dans notre étude est comparable avec la moyenne haute des taux d'intubation retrouvés dans la littérature dans le même contexte.

Dans l'enquête " 3 jours » réalisée par la SFAR en 1996, sur environ 300 anesthésies générales réalisées en fin d'accouchement (pour révision utérine ou délivrance artificielle), une intubation orotrachéale était réalisée dans seulement $8 \%$ des $\operatorname{cas}^{52}$. Les procédures anesthésiques n'étaient pas détaillées.

En 1997, une étude canadienne nationale déclarative a montré qu'en cas d'anesthésie générale pour délivrance artificielle chez des patientes stables, le taux d'intubation était d'environ $18 \%{ }^{55}$.

En 2000 une étude israélienne s'est intéressée au risque d'inhalation chez les patientes ayant bénéficié d'une anesthésie générale sans intubation trachéale au cours ou en fin de travail ${ }^{53}$. Dans cette étude rétrospective, sur les 2408 anesthésies générales pratiquées entre 1979 et 1993, seules $3,6 \%$ des patientes étaient intubées car, d'après les praticiens, celles-ci présentaient un risque élevé d'inhalation (RGO, jeûne de moins de 8 heures). De même que dans notre étude, toutes ces patientes avaient été intubées selon une induction en séquence rapide. Parmi les patientes non intubées, seule l'une d'elles a présenté un syndrome d'inhalation, non compliqué (sans pneumopathie).

Une étude plus récente, rétrospective, monocentrique, réalisée au CHU de Marseille entre 2005 et 2007, retrouvait un taux d'intubation de 5\%, sur les 111 anesthésies générales réalisées en fin de travail $^{48}$. Cinq des 6 patientes intubées avaient bénéficié d'une induction en séquence rapide. Les agents anesthésiques utilisés dans ce cas étaient le thiopental et la succinylcholine. La sixième patiente avait été intubée sous propofol et kétamine. Les 105 autres patientes avaient bénéficié d'une anesthésie générale en ventilation spontanée au masque, et les agents anesthésiques utilisés étaient le propofol et la kétamine. Aucune complication n'avait été recensée dans cette cohorte de patientes. Par la suite, une évaluation des pratiques a été menée dans le même centre, sous forme de questionnaires ${ }^{54}$. Sur 420 anesthésistes interrogés, 24\% déclaraient pratiquer une intubation orotrachéale systématiquement en cas de révision utérine ou de délivrance artificielle, soit un pourcentage comparable à celui de notre étude - qui était de $17 \%$ en cas de révision utérine et de $20 \%$ en cas de délivrance artificielle. En revanche, $45 \%$ déclaraient pratiquer systématiquement une intubation oro-trachéale en cas de manœuvres instrumentales, ce qui diffère de notre étude dans 
laquelle $100 \%$ des patientes étaient intubées en cas d'extraction fœtale. En cas d'intubation, une séquence rapide était réalisée par $82 \%$ des anesthésistes interrogés versus $100 \%$ dans notre étude.

\subsubsection{Intubation : la réticence des anesthésistes}

Le taux d'intubation retrouvé dans notre étude, et comparable aux données de la littérature, peut sembler faible par rapport au risque d'inhalation qui pourrait laisser présager d'un taux plus élevé d'intubation. Le fait que, dans la majorité des cas, l'anesthésiste ait privilégié la ventilation au masque montre que les risques liés à l'intubation (anaphylaxie et intubation/ventilation difficile) ont été évalués comme étant supérieurs au risque d'inhalation (plus important en cas de ventilation au masque).

Ceci s'explique d'une part par les facteurs physiologiques et anatomiques précédemment décrits qui peuvent rendre l'intubation plus difficile chez la femme enceinte, et d'autre part par les conditions de travail en salle de naissance. En effet, l'environnement en salle de naissance est moins adapté pour l'anesthésiste que l'environnement du bloc opératoire où tout le matériel est disponible, où l'espace est plus important et le personnel plus nombreux. En salle de naissance l'accès à la patiente (et notamment à la tête) est plus difficile, le monitorage est plus limité, et le matériel est beaucoup plus restreint (en particulier, absence de ventilateur). Ainsi, si une situation d'intubation/ventilation difficile se présente en salle de naissance, celle-ci sera plus délicate à gérer qu'au bloc opératoire ${ }^{56}$.

D’autre part, bien que le taux d'inhalation retrouvé dans notre étude soit plus élevé que celui retrouvé dans la littérature $\left(1 / 2000\right.$ à $\left.1 / 8000^{22}\right)$, il est important de noter que cette complication est survenue chez deux patientes qui avaient pourtant été chacune intubées. De plus, si l'on s'intéresse à l'ensemble des études de pratique sur le sujet, le taux d'inhalation recensé reste très faible, puisque en plus des deux complications de notre série, seule une inhalation était survenue dans l'étude israélienne parue en 2000, chez une patiente non intubée ${ }^{53}$. Ces différents éléments peuvent donc également expliquer en partie la réserve des anesthésistes vis-à-vis de l'intubation dans ce contexte, puisque l'analyse des pratiques actuelles (où l'intubation est loin d'être systématique) ne semble pas montrer, chez les patientes non intubées, un taux d'inhalation supérieur à celui retrouvé dans la littérature. De plus, comme le montre notre série, l'intubation ne semble pas protéger totalement du risque d'inhalation.

Cependant, ces éléments doivent être interprétés avec une grande prudence en raison des faibles effectifs des études réalisées et de la relative rareté de cette complication, qui rendent difficile la 
mise en évidence d'un risque d'inhalation significativement supérieur en l'absence d'intubation. De plus, on peut penser que l'inhalation survenue chez les deux patientes intubées aurait eu lieu même si ces patientes n'avaient pas été intubées, mais que les conséquences auraient été plus graves.

Enfin, l'intubation en fin de travail implique l'utilisation d'un curare (systématiquement dans notre étude et quasi-systématique dans l'ensemble de la littérature), ce qui a pour conséquence une majoration du risque anaphylactique par rapport à une anesthésie générale avec ventilation au masque facial, qui ne requiert pas de curare ${ }^{57}$. Ce risque anaphylactique est bien souligné par les deux derniers rapports du CNEMM de 2001 à 2006 et de 2007 à 2009: en effet l'anaphylaxie prédominait nettement dans les causes de décès liés à l'anesthésie puisqu'elle concernait au minimum 4 des 10 décès survenus sur ces deux périodes. Cet élément peut donc également inciter l'anesthésiste à opter pour une anesthésie générale sans curare, bien que dans notre étude la technique choisie n'était pas corrélée aux éventuels antécédents allergiques des patientes. Cette absence de corrélation concorde avec les études épidémiologiques sur l'anaphylaxie peranesthésique, montrant l'absence de lien entre un terrain allergique préexistant et la survenue d'une réaction allergique aux curares ${ }^{58}$.

Mais, même s'il semble pertinent de tenir compte de ce risque anaphylactique pour la réalisation d'une anesthésie générale en fin de travail, il est important de rappeler qu'en anesthésie, la mortalité liée à l'inhalation reste supérieure à celle liée à l'anaphylaxie (39 décès versus 14 en 1999) ${ }^{18}$. Néanmoins, ces données épidémiologiques proviennent d'études portant sur la population générale, et devraient donc être mises en balance avec les données issues spécifiquement de cohortes de patientes en fin de grossesse.

Pour conclure, la prise en compte prioritaire des risques liés à l'intubation par rapport au risque d'inhalation est confortée par les données de la littérature qui s'intéressent à la mortalité maternelle liée à l'anesthésie, en peri-partum. Ainsi, en France, les deux derniers rapports du CNEMM de 2001 à 2006 et de 2007 à 2009 ayant expertisé 6 dossiers sur les 10 patientes décédées d'une complication anesthésique au cours de ces deux périodes ont montré que 4 décès étaient liés à un problème anaphylactique, 1 à des difficultés d'intubation, et seulement 1 à une inhalation.

\subsubsection{Intubation : les facteurs déterminants}

Malgré ces différents éléments, le faible taux d'intubation retrouvé dans notre étude et dans la littérature ne peut être rattaché qu'aux réticences des anesthésistes par rapport à l'intubation. La 
technique de prise en charge des voies aériennes supérieures semble en effet résulter d'une réflexion approfondie, faite au cas par cas.

En effet, l'analyse des facteurs corrélés à l'intubation montre bien que certaines situations ou certains critères sont déterminants dans le choix de la technique anesthésique.

En premier lieu, c'est le geste obstétrical réalisé qui semble entrer en compte dans la décision de l'anesthésiste. En effet, les résultats de notre étude montraient par exemple que les patientes pour lesquelles une extraction fœtale était réalisée étaient systématiquement intubées alors que celles ayant une révision utérine n'étaient intubées que dans $20 \%$ des cas et celles bénéficiant d'une simple suture périnéale n'étaient jamais intubées. Il semble donc que plus le geste réalisé est invasif, plus l'intubation est fréquente. L'extraction fœtale représente ici le geste le plus invasif (par rapport à une simple DA ou RU), entraînant des compressions intra-abdominales importantes qui génèrent ellesmêmes des pics de pression intra-gastrique et majorent donc le risque d'inhalation. De plus il s'agit de la seule situation où l'anesthésie générale est réalisée alors que l'utérus est toujours gravide. Cela constitue également un facteur déterminant en faveur de l'intubation puisque la situation s'apparente alors au cas de l'anesthésie générale pour césarienne, pour laquelle la technique de référence est l'induction en séquence rapide ${ }^{59}$. A l'inverse, dès l'expulsion du fœtus, on observe une chute de la pression intra-gastrique et donc une augmentation du gradient pression intragastrique/pression œsophagienne, qui constitue alors une protection contre le risque d'inhalation ${ }^{60}$.

Deuxièmement, c'est le contexte clinique qui joue un rôle déterminant dans le choix de la technique anesthésique. En effet, les patientes présentant une hémorragie du post-partum sont significativement plus intubées que les autres. Cela s'explique probablement par la sécurité qu'apporte une intubation chez ces patientes qui peuvent rapidement évoluer vers un état de choc hémorragique, auquel cas l'intubation est nécessaire ${ }^{61}$. De plus, ces situations d'urgence requièrent la mise en œuvre de différentes actions dans le délai le plus bref possible (commande de produits dérivés du sang, conditionnement, prélèvements répétés, expansion volémique rapide, mise en place de thérapeutiques spécifiques... $)^{62}$. Or, on conçoit bien que la gestion d'une patiente intubée est plus simple que celle d'une patiente dont il faut surveiller, et assister si besoin, la ventilation.

Troisièmement, le terrain des patientes influence probablement également la décision de l'anesthésiste. En effet, on remarque que les patientes présentant un RGO ont tendance à être plus fréquemment intubées (on peut penser que la différence aurait été significative en analyse multivariée avec un effectif de patientes plus important et/ou moins de données manquantes), et que celles présentant un $I M C \geq 35 \mathrm{~kg} / \mathrm{m}^{2}$ sont significativement plus intubées que les autres. En ce 
qui concerne le RGO, de nombreuses études suggèrent qu'il serait un facteur de risque d'inhalation et qu'il devrait donc être pris en considération pour le choix de la technique anesthésique ${ }^{30,63}$. Ceci explique donc que les patientes présentant un RGO et cumulant donc au moins deux facteurs de risque d'inhalation (le RGO s'ajoutant au contexte de la grossesse) aient, en conséquence, plus tendance à être intubées. Concernant les patientes ayant un $I M C \geq 35 \mathrm{~kg} / \mathrm{m}^{2}$, il peut sembler paradoxal qu'elles soient plus fréquemment intubées, puisque ce critère constitue un des principaux facteurs de risque d'intubation difficile et devrait donc plutôt inciter à limiter les indications de I'intubation. C'est d'ailleurs le cas pour les autres facteurs prédictifs d'intubation difficile puisque, si I'on exclut les patientes ayant un $I M C \geq 35 \mathrm{~kg} / \mathrm{m}^{2}$, les patientes à risque d'intubation difficile ont bien tendance à être moins fréquemment intubées que les autres (14\% vs $19 \%)$. Ce paradoxe peut s'expliquer par le fait que chez les patients obèses la prévalence du RGO est supérieure à celle de la population générale, et notamment chez la femme ${ }^{64}$. Ce serait donc plutôt la présence d'un RGO, ou la forte suspicion de RGO chez les patientes obèses morbides, qui conduiraient à les intuber plus fréquemment (même si en analyse multivariée le RGO n'apparaissait pas comme un facteur associé de façon significative à l'intubation, probablement par manque de données). Une autre hypothèse possible pour expliquer ce paradoxe serait que l'anesthésiste craint, en cas de perte de la ventilation spontanée, des difficultés de ventilation au masque chez ces patientes obèses (puisque l'IMC > 26 $\mathrm{kg} / \mathrm{m}^{2}$ constitue un facteur prédictif de ventilation au masque difficile $\left.{ }^{14}\right)$, et préfère donc assurer l'accès aux voies aériennes supérieures par le biais d'une intubation oro-trachéale.

Enfin, en analyse univariée, le site du CHU de Rouen apparaissait clairement comme étant associé à un plus fort taux d'intubation. En analyse multivariée, cette association n'est plus qu'une tendance, mais qui serait peut-être confirmée par une étude plus puissante. Cette tendance à l'intubation plus fréquente au CHU pourrait être liée à son recrutement particulier, puisqu'on y prend en charge plus de patientes à risque que dans les $\mathrm{CH}$ périphériques. Elle pourrait aussi s'expliquer par le fait que la prise en charge au CHU implique certaines particularités. Notamment, une partie des praticiens travaillant au sein d'un CHU sont de jeunes médecins (chefs de clinique, assistants), ayant une expérience clinique moindre par rapport à celle de praticiens hospitaliers, habitués aux particularités de l'anesthésie obstétricale. Cette expérience plus limitée incite probablement à utiliser de préférence une technique a priori "plus sûre" dans ce contexte, à savoir l'intubation. De plus l'importance accordée à l'enseignement, caractéristique d'un $\mathrm{CHU}$, encourage peut-être les praticiens séniors à montrer aux plus jeunes la technique anesthésique de référence pour la femme enceinte qui reste l'intubation (même si ce travail montre que ce dogme ne s'applique peut-être pas dans ce contexte particulier qu'est l'anesthésie générale en fin de travail). 


\subsubsection{Séquences d'induction}

L'analyse des différentes techniques anesthésiques mises en œuvre au cours de l'anesthésie générale chez les patientes de la cohorte, montre une grande disparité des pratiques. En effet, pas moins de 14 associations différentes d'agents anesthésiques étaient recensées. Cela reflète bien l'absence de recommandation, et donc de consensus, concernant la gestion de l'anesthésie générale dans le cas précis de l'anesthésie générale en fin de travail. C'est notamment en cas d'anesthésie générale en ventilation spontanée que les associations d'agents anesthésiques utilisés sont les plus variables.

En revanche, $100 \%$ des patientes intubées avaient bénéficié d'une induction en séquence rapide, ce qui montre bien que le risque d'inhalation est largement considéré par les praticiens dans cette population de patientes. En effet, lorsque l'indication d'une intubation est posée, c'est finalement la technique de référence chez la femme enceinte (l'induction en séquence rapide) qui est systématiquement appliquée.

Il semble donc qu'il n'y ait pas de consensus sur la technique anesthésique idéale dans cette situation particulière qu'est l'anesthésie générale en fin de travail. Mais il apparaît clairement que le risque d'inhalation reste présent dans l'esprit des praticiens puisque lorsque c'est l'intubation qui est choisie, l'induction en séquence rapide - technique adaptée aux patients à risque d'inhalation - fait l'unanimité. L'utilisation large de la kétamine dans ce contexte va également dans ce sens puisque, lors d'une anesthésie générale en ventilation spontanée, il s'agit de l'agent anesthésique privilégié pour conserver au mieux les réflexes de protection des voies aériennes supérieures ${ }^{50}$.

\subsection{Limites de l'étude}

\subsubsection{Schéma de l'étude}

La principale limite de notre étude réside dans sa conception même puisqu'il s'agissait d'une étude rétrospective, de relativement faible effectif. Par conséquent, les résultats doivent être interprétés avec prudence, notamment en ce qui concerne les complications dont la fréquence de survenue est rare, et qui seront donc difficilement mises en évidence dans une petite cohorte. Le caractère rétrospectif de l'étude est inévitablement source de biais. Cependant une étude prospective n'aurait probablement pas permis de décrire de manière objective les pratiques courantes, en influençant les praticiens à pratiquer plus fréquemment l'intubation (toujours dans le cadre du respect du dogme de l'intubation systématique chez la femme enceinte). 


\subsubsection{Biais}

Les principaux biais de cette étude sont les biais de recrutement et de confusion. En ce qui concerne le biais de recrutement, il est lié au fait que plus de la moitié des patientes étaient prises en charge dans un $\mathrm{CHU}$, ce qui n'est pas représentatif de la population générale. Ce biais était en partie compensé par le fait que l'autre moitié des patientes étaient prises en charge dans un $\mathrm{CH}$ périphérique.

Lors de l'analyse des facteurs influençant le choix de la technique anesthésique, les biais de confusion ont été limités grâce à la réalisation d’une analyse multivariée.

\subsubsection{Données manquantes}

Une autre limite de notre étude concerne les données manquantes. Comme toute enquête rétrospective, l'analyse des données reposait essentiellement sur la qualité de la tenue des dossiers médicaux et notamment des feuilles d'anesthésie et des partogrammes. Trois éléments importants n’ont pu être analysés de façon concluante, en raison de certaines données manquantes.

Tout d'abord, nous pensons que la fréquence du RGO était sous-estimée. En effet, elle n'était que de $19 \%$ dans l'ensemble de la cohorte alors que, dans la littérature on retrouve une fréquence du RGO chez la femme enceinte allant de 30 à $50 \%$, voire jusqu'à $80 \%$ dans certaines études ${ }^{65}$. Cette sousestimation est due au fait que le RGO n'est probablement pas toujours recherché par les anesthésistes lors de la consultation d'anesthésie. Une recherche plus systématique de cette pathologie concluraient certainement à une augmentation de sa fréquence et donc peut-être à une augmentation du taux d'intubation.

Le second élément n'ayant pu faire l'objet d'une analyse pertinente en raison du manque de données, était l'administration d'un tampon de l'acidité gastrique. En effet, ce point pourtant essentiel dans la prévention du syndrome de Mendelson, n'était que très rarement mentionné sur les feuilles d'anesthésie ( $18 \%$ des cas). Il paraît évident que ce chiffre sous-estime largement les pratiques réelles. Le fait que ces traitements soient généralement administrés par la sage-femme et non par l'anesthésiste, pourrait expliquer l'absence fréquente de cette information sur la feuille d'anesthésie (par omission du médecin). Pour preuve, au CH des Feugrais où l'administration d'un tampon de l'acidité gastrique était la plus fréquente (64\% des cas), l'information figurait sur le partogramme (renseigné par les sages-femmes) et non sur la feuille d'anesthésie dans la totalité des 
dossiers. Cette donnée ne nous a donc pas paru interprétable, même si, pourtant, il nous semblait indispensable de s'assurer que le tamponnement de l'acidité gastrique était systématiquement réalisé avant une anesthésie générale dans ce contexte.

Enfin, le dernier élément important n'ayant pu être pris en considération était la durée du jeûne préanesthésique. Cette information, très probablement recueillie oralement par les anesthésistes auprès des patientes, n'était jamais mentionnée dans les dossiers. Ce critère est pourtant décisif dans le choix de la technique anesthésique employée, d'autant que le jeûne strict pendant le travail est actuellement discuté (cf chapitre 1.4.3.1) et que par conséquent les patientes ne sont pas systématiquement à jeun au moment de l'accouchement. Nous n'avons donc pas pu évaluer quelle était la place de ce critère dans la décision d'intuber, ou non, les patientes. Les différentes études retrouvées dans la littérature sur ce sujet n'avaient pas non plus pris en considération la durée du jeûne pré-anesthésique bien que, dans le cadre d'une anesthésie générale en fin de travail, ce paramètre doive nécessairement entrer en compte pour l'anesthésiste dans l'analyse de la balance bénéfice-risque.

\subsubsection{Place de l’anesthésie loco-régionale}

Du fait du caractère rétrospectif de cette étude, nous n'avons pu déterminer pour quelle(s) raison(s) une anesthésie loco-régionale n'avait pu être réalisée chez les patientes de la cohorte. En dehors des situations où les patientes présentent une contre-indication à l'anesthésie loco-régionale (en générale la rachianesthésie dans ce contexte), celle-ci devrait être systématiquement proposée puisqu'elle permet de s'affranchir des complications liées à l’anesthésie générale, quelles qu'elles soient ${ }^{1,42}$. Dans notre cohorte, on peut penser qu'une partie des patientes présentait une instabilité hémodynamique liée à une hémorragie du post-partum, contre-indiquant l’anesthésie périmédullaire. Mais pour les autres patientes, il est légitime de se demander si une technique alternative à l'anesthésie générale avait été envisagée, et si oui, pourquoi elle n'avait pu être mise en œuvre. Il aurait effectivement été intéressant de savoir, avant toute analyse des résultats, dans quelle proportion de cas l'anesthésie générale aurait pu être évitée, mais cette information ne figurait dans aucun des dossiers analysés (hormis pour les cas d’hémorragie grave du post-partum pour lesquelles l'anesthésie générale se justifiait aisément). 


\subsection{Intérêts de l'étude}

L'intérêt principal de notre étude était l'analyse des facteurs influençant le choix de la technique anesthésique. En effet aucune étude, à notre connaissance, n'avait jusqu'alors cherché à déterminer s'il existait un rationnel concernant le choix d'une anesthésie avec intubation versus une anesthésie en ventilation spontanée. Cette analyse nous a permis de mettre en évidence que ce choix était le fruit d'une réflexion faite au cas par cas, et qui tenait compte à la fois du geste réalisé, du contexte clinique et du terrain des patientes. II nous semblait essentiel de montrer qu'il y avait bien une démarche réfléchie lorsqu'il s'agissait d'intuber ou non une patiente lors d'une anesthésie générale en fin de travail, et qu'on ne pouvait se contenter d'évoquer un simple écart par rapport à ce qui pourrait être considéré, de prime abord, comme « les bonnes pratiques».

Par ailleurs, l'effectif relativement conséquent de l'étude et son caractère multicentrique en font une étude qui peut venir s'ajouter aux quelques autres s'étant déjà intéressées à ce sujet, permettant ainsi de renforcer le constat qu'il existe une grande disparité de pratique entre les différentes équipes. Cette disparité traduit l'absence de recommandation précise de la part des sociétés savantes sur ce sujet. C'est probablement grâce à la multiplicité d'études comme celle-ci que des recommandations pourront être élaborées.

\subsection{Perspectives}

On pourrait tout d'abord envisager la réalisation d'une enquête de pratique par questionnaire, spécifiquement axée sur les paramètres entrant en compte dans la démarche décisionnelle de l'anesthésiste dans le cas d'une anesthésie générale en fin de travail (durée du jeûne préanesthésique, contre-indication éventuelle à I'ALR, geste réalisé, terrain des patientes, contexte clinique, etc). Cela permettrait d'approfondir l'étude des facteurs déterminants dans le choix de la technique anesthésique et, éventuellement, de faire ressortir certains paramètres qui n'apparaissaient pas dans notre étude.

A terme, on peut penser que seule une étude prospective de grande ampleur, réalisée à l'échelle nationale, permettrait d'avoir une puissance suffisante pour conclure de façon fiable quant aux bénéfices mais surtout aux risques de chaque technique anesthésique, en particulier concernant l’anesthésie générale en ventilation spontanée. 
En fonction des conclusions d'une telle étude (ajoutées à celles des études de pratique préexistantes) on pourrait envisager la réalisation d'un algorithme spécifique aidant au choix de la technique anesthésique la plus adaptée à chaque cas, selon les éléments suivants : geste réalisé, contexte clinique, terrain de la patiente, durée du jeûne pré-anesthésique.

Voici quels seraient les points-clés d'un tel algorithme :

Avant tout, il est fondamental de rappeler qu'en l'absence de contre-indication, l'anesthésie locorégionale doit être pratiquée en première intention, puisqu'elle permet de s'affranchir des difficultés de gestion des voies aériennes supérieures.

Dans le cas où l'anesthésie générale ne peut être évitée, 3 situations bien spécifiques et imposant une prise en charge particulière semblent se distinguer :

1) L'anesthésie générale pour un geste simple chez une patiente stable, sans terrain particulier (type DA ou RU, sans hémorragie grave du post-partum), pour laquelle l'anesthésie générale en ventilation spontanée semble être un choix raisonnable.

2) L'anesthésie générale pour extraction instrumentale, pour laquelle l'intubation semble s'imposer.

3) L'anesthésie générale dans un contexte particulier : hémorragie grave du post-partum, RGO symptomatique, obésité morbide, prise alimentaire récente..., pour laquelle l'intubation doit probablement être réalisée, ou au moins discutée au cas par cas.

Dans tous les cas, la prophylaxie médicamenteuse du syndrome d'inhalation par l'administration d'un tampon de l'acidité gastrique est indispensable. 


\section{Conclusion}

Alors que l'anesthésie générale chez la femme enceinte fait l'objet de recommandations précises, le cas particulier de l'anesthésie générale en fin de travail est au contraire sujet à controverse. Si la gestion des voies aériennes supérieures est au centre du débat, c'est parce qu'elle constitue un enjeu majeur en terme de morbi-mortalité maternelle imputable à l'anesthésie. Or dans cette situation, l'anesthésiste est confronté à une double problématique, puisqu'il doit non seulement tenir compte du risque d'inhalation mais également des risques associés à l'intubation, tels que l'intubation difficile/impossible ou l'allergie aux curares. La complexité du choix de la technique anesthésique la plus adaptée résulte donc du fait que ce choix repose sur l'analyse d'une balance risque/risque, peutêtre plus que sur celle d’une balance bénéfice/risque.

Cette étude de pratique a permis de souligner quelques points essentiels de la prise en charge anesthésique des patientes en fin de travail; elle a également permis d'élaborer une ébauche d'algorithme en fonction de différents éléments-clés, tels que : la nature du geste réalisé, le terrain de la patiente, le contexte clinique, la durée du jeûne pré-anesthésique.

L'application d'un tel algorithme pourrait permettre une harmonisation des pratiques et, surtout, offrir un cadre aux praticiens exerçant en maternité et amenés à gérer l'anesthésie générale en fin de travail. On légitimerait ainsi une pratique largement utilisée (l'anesthésie générale en ventilation spontanée), mais encore considérée, à ce jour, par certains, comme un écart par rapport "aux bonnes pratiques » et au dogme de l'intubation systématique chez la femme enceinte.

Toutefois, le manque de puissance de notre étude doit inciter à considérer avec prudence l'innocuité de l'anesthésie générale en ventilation spontanée. 


\section{Références bibliographiques}

1. Boutonnet M, Faitot V, Keïta H. [Airway management in obstetrics]. Ann Fr Anesth Reanim. 2011 Sep;30(9):651-64.

2. Diemunsch P, Mercier F-J, Noll E. [Obstetric anaesthesia for instrumental vaginal delivery]. J Gynecol Obstet Biol Reprod (Paris). 2008 Dec;37 Suppl 8:S269-275.

3. Dailland P. Protocoles d'anesthesie-reanimation obstetricale. [S.I.]: Arnette Blackwell;

4. Hawthorne L, Wilson R, Lyons G, Dresner M. Failed intubation revisited: 17-yr experience in a teaching maternity unit. Br J Anaesth. 1996 May 1;76(5):680-4.

5. Vanner RG, Goodman NW. Gastro-oesophageal reflux in pregnancy at term and after delivery. Anaesthesia. 1989 Oct;44(10):808-11.

6. Wong CA, Loffredi M, Ganchiff JN, Zhao J, Wang Z, Avram MJ. Gastric emptying of water in term pregnancy. Anesthesiology. 2002 Jun;96(6):1395-400.

7. O'Sullivan GM, Sutton AJ, Thompson SA, Carrie LE, Bullingham RE. Noninvasive measurement of gastric emptying in obstetric patients. Anesth Analg. 1987 Jun;66(6):505-11.

8. Bogod DG. The postpartum stomach--when is it safe? Anaesthesia. 1994 Jan;49(1):1-2.

9. Boulay, G., \& Hamza, J. (2002). Anesthésie et estomac plein. 44e congrès national d'anesthésie et de réanimation, 217-26.

10. Blondel B, Supernant K, du Mazaubrun C, Bréart G. La santé périnatale en France métropolitaine de 1995 à 2003: Résultats des enquêtes nationales périnatales. Journal de Gynécologie Obstétrique et Biologie de la Reproduction. 2006 Jun;35(4):373-87.

11. Juvin P, Lavaut E, Dupont H, Lefevre P, Demetriou M, Dumoulin J-L, et al. Difficult Tracheal Intubation Is More Common in Obese Than in Lean Patients. Anesth Analg. 2003 Aug 1;97(2):595-600.

12. Saravanakumar K, Rao SG, Cooper GM. Obesity and obstetric anaesthesia. Anaesthesia. 2006;61(1):36-48.

13. Izci B, Riha RL, Martin SE, Vennelle M, Liston WA, Dundas KC, et al. The upper airway in pregnancy and pre-eclampsia. Am J Respir Crit Care Med. 2003 Jan 15;167(2):137-40.

14. Diemunsch P, Langeron O, Richard M, Lenfant F. [Prediction and definition of difficult mask ventilation and difficult intubation: question 1. Société Française d'Anesthésie et de Réanimation]. Ann Fr Anesth Reanim. 2008 Jan;27(1):3-14.

15. Rapport du Comité national d'experts sur la mortalité maternelle (CNEMM) 2001-2006. Unité Inserm 953, Institut de veille sanitaire. Janvier 2010, 102 pages.

16. Bouvier-Colle MH, Péquignot $F$, Jougla E. [Maternal mortality in France: frequency, trends and causes]. J Gynecol Obstet Biol Reprod (Paris). 2001 Dec;30(8):768-75. 
17. Saucedo $M$, Deneux-Tharaux C, Bouvier-Colle M-H. Épidémiologie de la mortalité maternelle en France, 2007-2009. Journal de Gynécologie Obstétrique et Biologie de la Reproduction. 2013 Nov;42(7):613-27.

18. Lienhart A, Auroy $Y$, Péquignot $F$, Benhamou D, Warszawski J, Bovet $M$, et al. Survey of anesthesia-related mortality in France. Anesthesiology. 2006 Dec;105(6):1087-97.

19. Deneux-Tharaux C, Carmona E, Bouvier-Colle M-H, Bréart G. Postpartum maternal mortality and cesarean delivery. Obstet Gynecol. 2006 Sep;108(3 Pt 1):541-8.

20. Mertes PM, De Blay F, Dong S. Risque allergique en anesthésie. La Presse Médicale. 2013 Mar;42(3):269-79.

21. Mendelson $\mathrm{Cl}$. The aspiration of stomach contents into the lungs during obstetric anesthesia. Am J Obstet Gynecol. 1946 Aug;52:191-205.

22. Chassard D, Mercier F-J. Quelles stratégies appliquer pour diminuer le risque d'une inhalation pulmonaire de liquide gastrique pendant une anesthésie générale ? Annales Françaises d’Anesthésie et de Réanimation. 2009 Mar;28(3):197-9.

23. Devos, N., and B. Dureuil. "Le syndrome d'inhalation." SFAR, Conférences d'actualisation. 42ième Congrès Société Française d'Anesthésie et de Réanimation, ed. Paris Elsevier (2000): 127-39.

24. Olsson GL, Hallen B, Hambraeus-Jonzon K. Aspiration during anaesthesia: a computer-aided study of 185,358 anaesthetics. Acta Anaesthesiol Scand. 1986 Jan;30(1):84-92.

25. Warner MA, Warner ME, Weber JG. Clinical significance of pulmonary aspiration during the perioperative period. Anesthesiology. 1993 Jan;78(1):56-62.

26. Neelakanta G, Chikyarappa A. A review of patients with pulmonary aspiration of gastric contents during anesthesia reported to the Departmental Quality Assurance Committee. J Clin Anesth. 2006 Mar;18(2):102-7.

27. Sakai T, Planinsic RM, Quinlan JJ, Handley LJ, Kim T-Y, Hilmi IA. The Incidence and Outcome of Perioperative Pulmonary Aspiration in a University Hospital: A 4-Year Retrospective Analysis: Anesthesia \& Analgesia. 2006 Oct;103(4):941-7.

28. Auroy $\mathrm{Y}$, Benhamou D, Péquignot F, Jougla E, Lienhart A. [Survey of anaesthesia-related mortality in France: the role of aspiration of gastric contents]. Ann Fr Anesth Reanim. 2009 Mar;28(3):200-5.

29. Cooper GM, McClure JH. Maternal deaths from anaesthesia. An extract from Why Mothers Die 2000-2002, the Confidential Enquiries into Maternal Deaths in the United Kingdom Chapter 9: Anaesthesia. Br J Anaesth. 2005 Apr 1;94(4):417-23.

30. Debaene, B. et Jeanny, A. Anesthésie pour estomac plein. Praticien en anesthésie réanimation, 2007, vol. 11, p. 1 S21.

31. Quinn AC, Milne D, Columb M, Gorton H, Knight M. Failed tracheal intubation in obstetric anaesthesia: 2 yr national case-control study in the UK. Br J Anaesth. 2013 Jan 1;110(1):74-80. 
32. Goldszmidt E. Is there a Difference Between the Obstetric and Non-obstetric Airway? In: Halpern SH, Douglas MJ, editors. Evidence-based Obstetric Anesthesia [Internet]. Blackwell Publishing Ltd; 2007 [cited 2013 Oct 13]. p. 225-36. Available from: http://onlinelibrary.wiley.com/doi/10.1002/9780470988343.ch19/summary

33. Samsoon GLT, Young JRB. Difficult tracheal intubation: a retrospective study. Anaesthesia. 1987;42(5):487-90.

34. McDonnell NJ, Paech MJ, Clavisi OM, Scott KL, ANZCA Trials Group. Difficult and failed intubation in obstetric anaesthesia: an observational study of airway management and complications associated with general anaesthesia for caesarean section. Int J Obstet Anesth. 2008 Oct;17(4):292-7.

35. Rocke DA, Murray WB, Rout CC, Gouws E. Relative risk analysis of factors associated with difficult intubation in obstetric anesthesia. Anesthesiology. $1992 \mathrm{Jul} ; 77(1): 67-73$.

36. Mallampati SR, Gatt SP, Gugino LD, Desai SP, Waraksa B, Freiberger D, et al. A clinical sign to predict difficult tracheal intubation: a prospective study. Can Anaesth Soc J. 1985 Jul;32(4):42934.

37. Pilkington S, Carli F, Dakin MJ, Romney M, Witt K a. D, DORé CJ, et al. Increase in Mallampati score during pregnancy. Br J Anaesth. 1995 Jun 1;74(6):638-42.

38. Kodali B-S, Chandrasekhar S, Bulich LN, Topulos GP, Datta S. Airway changes during labor and delivery. Anesthesiology. 2008 Mar;108(3):357-62.

39. Blondel B, Kermarrec M. Enquête nationale périnatale 2010. In: Les naissances en 2010 et leur évolution depuis 2003. Paris: Inserm; 2011, http://www.sante.gouv.fr.

40. Hawkins JL, Chang J, Palmer SK, Gibbs CP, Callaghan WM. Anesthesia-related maternal mortality in the United States: 1979-2002. Obstet Gynecol. 2011 Jan;117(1):69-74.

41. Bouvet, L., \& Benhamou, D. (2008). Les règles du jeûne préopératoire. Le Praticien en Anesthésie Réanimation, 12(6), 413-421.

42. American Society of Anesthesiologists Task Force on Obstetric Anesthesia. Practice guidelines for obstetric anesthesia: an updated report by the American Society of Anesthesiologists Task Force on Obstetric Anesthesia. Anesthesiology. 2007 Apr;106(4):843-63.

43. Merchant R, Chartrand D, Dain S, Dobson G, Kurrek M, Lagacé A, et al. Guidelines to the practice of anesthesia revised edition 2013. Can J Anaesth. 2013 Jan;60(1):60-84.

44. Société française d'anesthésie et de réanimation. Les blocs périmédullaires chez l'adulte. Recommandations pour la pratique clinique. 2006 http://www.sfar.org/_docs/articles/rpc_perimedullaire.pdf.

45. Diemunsch $P$, Haliska W, Szczot M, Noudem Y. [Eating during labour: is it of any benefit?]. Ann Fr Anesth Reanim. 2006 Jun;25(6):609-14.

46. Scrutton MJ, Metcalfe GA, Lowy C, Seed PT, O'Sullivan G. Eating in labour. A randomised controlled trial assessing the risks and benefits. Anaesthesia. 1999 Apr;54(4):329-34. 
47. Paranjothy S, Griffiths JD, Broughton HK, Gyte GM, Brown HC, Thomas J. Interventions at caesarean section for reducing the risk of aspiration pneumonitis. Cochrane Database Syst Rev. 2010;(1):CD004943.

48. Zieleskiewicz L, Bellefleur J-P, Antonini F, Ortega D, Leone M, Martin C. [Airway management for anaesthesia performed at the end of labour: survey of practices]. Ann Fr Anesth Reanim. 2009 Feb;28(2):119-23.

49. Dureuil, B. La ventilation spontanée en anesthésie. Conférence d'actualisation de la SFAR;

50. Laryngeal Competence with Ketamine and Other Drugs : Anesthesiology [Internet]. [cited 2013 Dec 24]. Available from:

http://journals.Iww.com/anesthesiology/Fulltext/1973/02000/Laryngeal_Competence_with_Ke tamine_and_Other_Drugs.5.aspx

51. Duflo, F., Allaouchiche, B., \& Chassard, D. (2000). Urgences anesthésiques obstétricales.

Conférences d'actualisation, 43.

52. Clergue $F$, Auroy $Y$, Péquignot $F$, Jougla $E$, Lienhart $A$, Laxenaire $M C$. French survey of anesthesia in 1996. Anesthesiology. 1999 Nov;91(5):1509-20.

53. Ezri T, Szmuk P, Stein A, Konichezky S, Hagai T, Geva D. Peripartum general anasthesia without tracheal intubation: incidence of aspiration pneumonia. Anaesthesia. 2000 May;55(5):421-6.

54. Zieleskiewicz L, Bellefleur JP, Leone M. [Upper airway management in obsterics: results of a French survey]. Can J Anaesth. 2009 Mar;56(3):265-6.

55. Breen TW, McNeil T, Dierenfield L. Obstetric anesthesia practice in Canada. Can J Anaesth. 2000 Dec;47(12):1230-42.

56. A. Steib, O. Collange. Anesthésie en dehors du bloc opératoire. 2008.

57. Mertes PM, Malinovsky JM, Jouffroy L, Working Group of the SFAR and SFA, Aberer W, Terreehorst I, et al. Reducing the risk of anaphylaxis during anesthesia: 2011 updated guidelines for clinical practice. J Investig Allergol Clin Immunol. 2011;21(6):442-53.

58. Mertes PM, Alla F, Tréchot $P$, Auroy $Y$, Jougla E, Groupe d'Etudes des Réactions Anaphylactoïdes Peranesthésiques. Anaphylaxis during anesthesia in France: an 8-year national survey. J Allergy Clin Immunol. 2011 Aug;128(2):366-73.

59. Wee MYK, Brown H, Reynolds F. The National Institute of Clinical Excellence (NICE) guidelines for caesarean sections: implications for the anaesthetist. Int J Obstet Anesth. 2005 Apr;14(2):147-58.

60. Hartsilver EL, Vanner RG, Bewley J, Clayton T. Gastric pressure during emergency caesarean section under general anaesthesia. Br J Anaesth. 1999 May;82(5):752-4.

61. Rossignol M, Figuet S, Benlolo S, Payen D. Prise en charge anesthésique des hémorragies du post-partum. In : Conférences d'actualisation de la SFAR 2004, Elsevier SAS 2004, pp 653-672.

62. Recommandations pour la Pratique Clinique - Hémorragies du post-partum immédiat. J Gynecol Obstet Biol Reprod (Paris) $2004 ; 33$ : Suppl N 8 . Également accessible sur le site Internet de la Haute Autorité de Santé : http://www.has-sante.fr/portail/display.jsp?id=c_268413. 
63. Landreau B, Odin I, Nathan N. Inhalation gastrique : épidémiologie et facteurs de risque. Annales Françaises d'Anesthésie et de Réanimation. 2009 Mar;28(3):206-10.

64. Nilsson M, Johnsen R, Ye W, Hveem K, Lagergren J. Obesity and estrogen as risk factors for gastroesophageal reflux symptoms. JAMA. 2003 Jul 2;290(1):66-72.

65. Richter JE. Gastroesophageal reflux disease during pregnancy. Gastroenterology Clinics of North America. 2003 Mar;32(1):235-61. 


\section{Résumé}

La gestion des voies aériennes en cas d'anesthésie générale (AG) en fin de travail, situation devenue relativement rare, ne fait l'objet d'aucune recommandation spécifique. II est cependant admis que l'intubation oro-trachéale (IOT) en séquence rapide est la règle chez la femme enceinte du fait du risque d'inhalation. Cependant il existe une discordance majeure entre ce dogme et les pratiques cliniques dans le cas spécifique de l'AG en fin de travail. Cet écart s'explique par le fait que l'intubation peut parfois être considérée comme plus à risque dans ce contexte qu'une anesthésie en ventilation spontanée. Dans l'enquête " 3 jours » réalisée par la SFAR ${ }^{1}$ en 1996 le taux d'IOT était seulement de $8 \%$ chez les 30000 parturientes nécessitant une AG en salle de naissance. En 2008, une enquête de pratique rétrospective réalisée au CHU de Marseille ${ }^{2}$, incluant 111 patientes, retrouvait un taux d'IOT dans seulement $5 \%$ des cas d'AG en fin de travail. Dans ces deux études aucune complication n'était survenue.

Afin d'évaluer l'état actuel des pratiques dans ce contexte, nous avons réalisé une étude en région HauteNormandie concernant la prise en charge des voies aériennes dans l'AG en fin de travail.

Il s'agissait d'une étude multicentrique (3 centres), observationnelle, rétrospective, réalisée de janvier 2009 à décembre 2010, incluant toutes les parturientes ayant nécessité une manœuvre obstétricale en fin d'accouchement sous AG (délivrance artificielle et/ou révision utérine, révision sous valves, extraction fœtale, ou suture périnéale). Le critère de jugement principal était le taux d'IOT. Les autres critères étudiés étaient le taux de complications et les facteurs influençant le choix de la techique anesthésique. Les données quantitatives sont exprimées sous forme de moyenne ( \pm écart type). Les données qualitatives sont exprimées en pourcentage.

Cent trente patientes ont été incluses, 68 au CHU de Rouen, 48 au $\mathrm{CH}$ du Belvédère et 14 au $\mathrm{CH}$ des Feugrais. Le taux global d'IOT était de $21 \%$ ( $31 \%$ au CHU de Rouen et $10 \%$ en moyenne dans les $\mathrm{CH}$ périphériques). Toutes les IOT ont été réalisées en séquence rapide. Les patientes non intubées bénéficiaient le plus souvent d'une anesthésie en ventilation spontanée au masque, par Kétamine et/ou Propofol.

Un seul épisode de vomissements sans inhalation et une probable pneumopathie d'inhalation sont survenus au cours des actes anesthésiques. Dans les deux cas il s'agissait de patientes intubées. Aucune IOT difficile, ni épisode de désaturation profonde n'a été rapporté.

L'analyse multivariée des facteurs pouvant influençer le choix de la technique anesthésique a montré que I'hémorragie du post-partum et l'IMC $\geq 35 \mathrm{~kg} / \mathrm{m}^{2}$ étaient associés à un plus fort taux d'intubation (odd ratio respectivement à 14,3 (Intervalle de confiance à 95\% [1,8-114,8] $(p=0,01)$ )et 6,1 (Intervalle de confiance à 95\% [1,3-28] $(p=0,02))$. A ces facteurs s'ajoute également l'extraction fœtale puisqu'elle était associée à une intubation dans $100 \%$ des cas $(p<0,0001)$.

Le taux d'IOT dans notre étude est comparable à ceux précédemment retrouvés dans la littérature. II persiste cependant un important écart avec le dogme de I'IOT systématique dès 14 semaines d'aménorrhé. Cet écart semble toutefois résulter d'une analyse approfondie, au cas par cas, de la balance risque/risque (AG avec intubation versus $A G$ en ventilation spontanée au masque), puisque certaines situations particulières étaient fortement associés à l'intubation.

Par ailleurs, les seules complications sont survenues lors d'une IOT. Pour autant, en raison du manque de puissance et du caractère retrospectif de cette étude, il n'est pas possible de conclure à l'innocuité de l'AG en ventilation spontanée, qui semble être devenue la règle dans ce contexte. Cette pratique mériterait donc d’être validée par une étude à grande échelle.

${ }^{1}$ Anesthesiology 1999; 91: 1509-20

${ }^{2}$ Ann Fr Anesth Reanim 2009; 28: 119-123

Mots-clés : anesthésie générale, obstétrique, salle de naissance, voies aériennes supérieure, intubation 